\title{
Integral Structure for Simple Singularities
}

\author{
Todor MILANOV and Chenghan ZHA
}

Kavli IPMU (WPI), UTIAS, The University of Tokyo, Kashiwa, Chiba 277-8583, Japan

E-mail: todor.milanov@ipmu.jp, chenghan.zha@ipmu.jp

Received May 27, 2020, in final form August 09, 2020; Published online August 22, 2020

https://doi.org/10.3842/SIGMA.2020.081

\begin{abstract}
We compute the image of the Milnor lattice of an ADE singularity under a period map. We also prove that the Milnor lattice can be identified with an appropriate relative $K$-group defined through the Berglund-Hübsch dual of the corresponding singularity.
\end{abstract}

Key words: simple singularities; period map; mirror symmetry; topological K-theory

2020 Mathematics Subject Classification: 14D05; 32S30; 19L47

\section{Introduction}

Let $f \in \mathbb{C}\left[x_{1}, x_{2}, x_{3}\right]$ be a weighted homogeneous polynomial representing the germ of a simple singularity of type $\mathrm{A}, \mathrm{D}$, or E. Let $f^{T} \in \mathbb{C}\left[x_{1}, x_{2}, x_{3}\right]$ be the corresponding Berglund-Hübsch dual of $f$ (see Section 1.2). Fan-Jarvis-Ruan proved in [6], using also results of Givental-Milanov [10] and Frenkel-Givental-Milanov [7], that the generating function of Fan-Jarvis-Ruan-Witten (FJRW) invariants of $f^{T}$ can be identified with a tau-function of a specific Kac-Wakimoto hierarchy. The identification however involves rescaling the dynamical variables of the KacWakimoto hierarchy and the precise values of the rescaling constants were left unknown. One application of the results in this papers is to obtain explicit formulas for the rescaling constants. Such an explicit identification is needed if one is interested in constructing a matrix model for the FJRW invariants of $f^{T}$, similar to the Kontsevich's matrix model in [13]. We are not going to compute the rescaling coefficients in this paper. The computation is straightforward and it should probably be done only when needed. Let us try to explain instead why this small technical detail leads to a very interesting problem in singularity theory.

Let us recall that for any singularity $f$ there is a natural way to construct a semi-simple Frobenius structure on the space of miniversal deformations of $f$ (see [11]). The construction depends on the choice of a primitive form in the sense of Saito [16] and it essentially coincides with what Saito called flat structure. On the other hand, motivated by Gromov-Witten theory, Givental introduced the notion of a total descendent potential for every semi-simple Frobenius manifold (see [8, 9]). Givental conjectured [9] and Teleman proved [19] that if the Frobenius structure corresponding to the quantum cohomology of a compact Kähler manifold $X$ is semisimple, then his definition coincides with the generating function of Gromov-Witten invariants of $X$. Let us return to our settings, i.e., the case of a simple weighted homogeneous singularity $f$ on 3 variables. The standard holomorphic volume form $d x_{1} \wedge d x_{2} \wedge d x_{3}$ is primitive. Therefore, following Givental, we can define total descendent potential. The latter will be called, the total descendent potential of $f$. Fan-Jarvis-Ruan proved in [6] that the generating function of FJRW invariants of $f^{T}$ coincides with the total descendant potential of $f$. Furthermore, GiventalMilanov [10] and Frenkel-Givental-Milanov [7] proved that the total descendant potential of $f$ is a tau-function of the principal Kac-Wakimoto hierarchy of the same type A, D, or E as the singularity $f$. Finally, the outcome of the above work is that the generating function of FJRW

This paper is a contribution to the Special Issue on Primitive Forms and Related Topics in honor of Kyoji Saito for his 77th birthday. The full collection is available at https://www.emis.de/journals/SIGMA/Saito.html 
invariants of $f^{T}$ is a tau-function of an appropriate Kac-Wakimoto hierarchy. However, there is still a small gap in this statement. Namely, while the state space of FJRW theory is identified explicitly with the Milnor ring of the singularity (see [6]), the identification of the Milnor ring and the Cartan subalgebra of the corresponding simple Lie algebra is given by a period map and it is not explicit. In order to obtain an explicit identification, we need to determine the image of the root lattice in the Milnor ring of the singularity. This is exactly the problem that we want to solve in this paper.

\section{$1.1 \quad$ Simple singularities}

Let us give a precise statement of the problem that we want to solve. Let $f\left(x_{1}, x_{2}, x_{3}\right)=$ $g\left(x_{1}, x_{2}\right)+x_{3}^{2}$, where $g$ is one of the polynomials listed in the following table:

\begin{tabular}{c|ccccc} 
Type & $A_{N}$ & $D_{N}$ & $E_{6}$ & $E_{7}$ & $E_{8}$ \\
\hline$g$ & $x_{1}^{N+1}+x_{2}^{2}$ & $x_{1}^{2} x_{2}+x_{2}^{N-1}$ & $x_{1}^{3}+x_{2}^{4}$ & $x_{1}^{3}+x_{1} x_{2}^{3}$ & $x_{1}^{3}+x_{2}^{5}$
\end{tabular}

The polynomial $f$ represents the germ of a simple singularity at $x=0$. Let

$$
H_{f}:=\mathbb{C}\left[x_{1}, x_{2}, x_{3}\right] /\left(f_{x_{1}}, f_{x_{2}}, f_{x_{3}}\right)
$$

be the Milnor ring of $f$, where $f_{x_{i}}:=\frac{\partial f}{\partial x_{i}}$. Let us denote by $($,$) the residue pairing on H_{f}$ corresponding to the standard volume form $\omega=d x_{1} \wedge d x_{2} \wedge d x_{3}$, that is,

$$
\left(\phi_{1}(x), \phi_{2}(x)\right):=\operatorname{Res}_{x=0} \frac{\phi_{1}(x) \phi_{2}(x) \omega}{f_{x_{1}} f_{x_{2}} f_{x_{3}}} .
$$

The hypersurfaces $V_{\lambda}=\left\{x \in \mathbb{C}^{3} \mid f(x)=\lambda\right\}$ for $\lambda \neq 0$ are non-singular and their union has a structure of a smooth fibration on $\mathbb{C} \backslash\{0\}$ known as the Milnor fibration. Let us fix a reference point $\lambda=1$ and consider the middle homology group $H_{2}\left(V_{1} ; \mathbb{Z}\right)$, known also as the Milnor lattice. Our interest is in the period vectors $I_{\alpha}^{(-1)}(\lambda) \in H_{f}$ defined by

$$
\left(I_{\alpha}^{(-1)}(\lambda), \phi_{i}\right):=\frac{1}{2 \pi} \int_{\alpha_{\lambda}} \phi_{i}(x) \frac{\omega}{d f},
$$

where $\alpha \in H_{2}\left(V_{1} ; \mathbb{C}\right), \phi_{i}(x)(1 \leq i \leq N)$ is a set of polynomials representing a basis of $H_{f}$, $\alpha_{\lambda} \in H_{2}\left(V_{\lambda} ; \mathbb{C}\right)$ is obtained from $\alpha$ via a parallel transport along some reference path, and $\frac{\omega}{d f}$ is the so-called Gelfand-Leray form (see [1]). Alternatively, we can view each period vector as a multivalued analytic function $I_{\alpha}^{(-1)}: \mathbb{C} \backslash\{0\} \rightarrow H_{f}$.

Let us assign degree $c_{i} \in \mathbb{Q}_{>0}$ to $x_{i}(1 \leq i \leq 3)$, such that, the polynomial $f$ has degree 1 . Then the Milnor ring becomes a graded ring. The highest possible degree of a homogeneous element in $H_{f}$ is $D=\sum_{i=1}^{3}\left(1-2 c_{i}\right)=1-\frac{2}{h}$, where $h$ is the Coxeter number of the corresponding root system. Put $\theta:=\frac{D}{2}-\operatorname{deg}$, where deg: $H_{f} \rightarrow H_{f}$ is the linear operator uniquely determined by the following condition: if $\phi$ is a weighted homogeneous element of degree $d$, then $\operatorname{deg}(\phi)=d \phi$. For homogeneity reasons, the period vectors have the form

$$
I_{\alpha}^{(-1)}(\lambda)=\frac{\lambda^{\theta+1 / 2}}{\Gamma(\theta+3 / 2)} \Psi(\alpha)
$$

where $\Psi: H_{2}\left(V_{1} ; \mathbb{C}\right) \rightarrow H_{f}$ is a linear isomorphism. Our goal is to compute the image of the Milnor lattice $H_{2}\left(V_{1} ; \mathbb{Z}\right)$ via the map $\Psi$. The solution to this problem is given in Section 2. Explicit formulas for the image of the Milnor lattice via the map $\Psi$ are given in Sections 2.3-2.7. The main feature of our answer is that it involves various $\Gamma$-constants and roots of unity. The second goal of our paper is to show that although the formulas look cumbersome, in fact there is an interesting structure behind them. 


\section{$1.2 \quad$ K-theoretic interpretation of the Milnor lattice}

It turns out that our answer can be stated quite elegantly via relative K-theory. The idea to look for such a description comes from the work of Iritani [12], Chiodo-Iritani-Ruan [4], and ChiodoNagel [5]. More precisely, Iritani was able to prove in [12] that the Milnor lattice of the mirror of a Fano toric orbifold $X$ can be identified with the topological K-ring $K^{0}(X)$. The identification uses a period map to embed the Milnor lattice in $H^{*}(X ; \mathbb{C})$ and a certain $\Gamma$-class modification of the Chern character map to embed $K^{0}(X)$ in $H^{*}(X ; \mathbb{C})$. The lattice in $H^{*}(X ; \mathbb{C})$, obtained either as the image of the Milnor lattice via the period map or as the image of $K^{0}(X)$ via the $\Gamma$-class modification of the Chern character map, is known as $\Gamma$-integral structure in quantum cohomology. Isolated singularities are almost never mirror models of a manifold. Nevertheless, Chiodo-Iritani-Ruan have proposed an analogue of the $\Gamma$-integral structure for singularities of Fermat type. The analogue of $H^{*}(X ; \mathbb{C})$ is played by the Milnor ring $H_{f}$, while $K^{0}(X)$ is replaced with an appropriate category of equivariant matrix factorizations of $f$. Finally, Chiodo-Nagel were able to find an isomorphism between $H_{f}$ and an appropriate relative orbifold cohomology group. Since, the Chern character gives an isomorphism between cohomology and K-theory and the Grothendieck group of the category of matrix factorization also has the flavor of a topological K-ring, after expecting more carefully the constructions in [4] and [5], we see that there is a natural candidate for a $\Gamma$-integral structure for Fermat type singularities. After several trial and errors we were able to find the correct topological $K$-ring and the correct modification of the Chern character map. Moreover our proposal makes sense not only for Fermat type polynomials, but more generally for an arbitrary invertible polynomial. Nevertheless, let us return to our current settings of simple singularities. We believe that our results can be generalized to all invertible polynomials, but that would require some additional work.

The polynomials $f$ corresponding to a simple singularity are invertible polynomials in the sense of [3] (see also [14]). Each polynomial is uniquely determined by a $3 \times 3$ matrix $A=$ $\left(a_{i j}\right)_{1 \leq i, j \leq 3}$ with non-negative integer coefficients, such that,

$$
f(x)=\sum_{i=1}^{3} x_{1}^{a_{i 1}} x_{2}^{a_{i 2}} x_{3}^{a_{i 3}} .
$$

Following Fan-Jarvis-Ruan (see [6]) we consider also the Berglund-Hübsch dual polynomial

$$
f^{T}(x)=\sum_{i=1}^{3} x_{1}^{a_{1 i}} x_{2}^{a_{2 i}} x_{3}^{a_{3 i}} .
$$

Let $G^{T}$ be the group of diagonal symmetries of $f^{T}$, that is,

$$
G^{T}:=\left\{t \in\left(\mathbb{C}^{*}\right)^{3} \mid t_{1}^{a_{1 i}} t_{2}^{a_{2 i}} t_{3}^{a_{3 i}}=1 \forall i\right\} .
$$

Let $a^{i j}(1 \leq i, j \leq 3)$ be the entries of the inverse matrix $A^{-1}$. The group $G^{T}$ is generated by the following elements

$$
\bar{\rho}_{i}=\left(e^{2 \pi \mathbf{i} a^{i 1}}, e^{2 \pi \mathbf{i} a^{i 2}}, e^{2 \pi \mathbf{i} a^{i 3}}\right), \quad 1 \leq i \leq 3 .
$$

Finally, let $V_{1}^{T}=\left\{x \in \mathbb{C}^{3} \mid f^{T}(x)=1\right\}$. Our main interest is in the topological relative Ktheoretic orbifold group

$$
K_{\text {orb }}^{0}\left(\left[\mathbb{C}^{3} / G^{T}\right],\left[V_{1}^{T} / G^{T}\right]\right):=K_{G^{T}}^{0}\left(\mathbb{C}^{3}, V_{1}^{T}\right) .
$$

In general, there is no satisfactory definition of K-theory for non-compact spaces. However, in our case the pair $\left(\mathbb{C}^{3}, V_{1}^{T}\right)$ is $G^{T}$-equivariantly homotopic to a pair of finite $C W$ complexes, so 
we may think of $\left(\mathbb{C}^{3}, V_{1}^{T}\right)$ as a $G^{T}$-equivariant pair of finite $C W$-complexes. We refer to [18] for some background on equivariant topological K-theory.

Motivated by Iritani's $\Gamma$-integral structure in quantum cohomology (see [12]), we will now construct a linear map

$$
\operatorname{ch}_{\Gamma}: K_{\text {orb }}^{0}\left(\left[\mathbb{C}^{3} / G^{T}\right],\left[V_{1}^{T} / G^{T}\right]\right) \otimes \mathbb{C} \longrightarrow H_{\text {orb }}\left(\left[\mathbb{C}^{3} / G^{T}\right],\left[V_{1}^{T} / G^{T}\right] ; \mathbb{C}\right),
$$

which is a certain $\Gamma$-class modification of the orbifold Chern character map. For a $G^{T}$-equivariant space $X$ and $g \in G^{T}$, let us denote by $\operatorname{Fix}_{g}(X):=\{x \in X \mid g x=x\}$ the set of fixed points. The elements in the relative $K$-group will be identified with isomorphism classes $[E \rightarrow F]$ of two-term complexes $E \stackrel{d}{\longrightarrow} F$ of $G^{T}$-equivariant vector bundles, such that, the differential $d$ is a morphism of $G^{T}$-equivariant vector bundles and $\left.d\right|_{V_{1}^{T}}: E_{V_{1}^{T}} \rightarrow F_{V_{1}^{T}}$ is an isomorphism. Note that for $g \in G^{T}$, the restriction of a vector bundle $\left.E\right|_{\text {Fix }_{g}\left(\mathbb{C}^{3}\right)}$ decomposes as a direct sum of eigen-subbundles $E_{\zeta}$ and that the restriction to Fix $_{g}\left(\mathbb{C}^{3}\right)$ of every two term complex $E \stackrel{d}{\longrightarrow} F$ decomposes as a direct sum of two term subcomplexes $E_{\zeta} \stackrel{d_{\zeta}}{\longrightarrow} F_{\zeta}$, where $d_{\zeta}=\left.d\right|_{E_{\zeta}}$. We have the following well known decomposition (e.g., see [2, Theorem 2]):

$$
\operatorname{Tr}: \quad K_{G^{T}}^{0}\left(\mathbb{C}^{3}, V_{1}^{T}\right) \otimes \mathbb{C} \stackrel{\cong}{\longrightarrow} \bigoplus_{g \in G^{T}}\left[K^{0}\left(\operatorname{Fix}_{g}\left(\mathbb{C}^{3}\right), \operatorname{Fix}_{g}\left(V_{1}^{T}\right)\right) \otimes \mathbb{C}\right]^{G^{T}},
$$

where [ $]^{G^{T}}$ denotes the $G^{T}$-invariant part and the morphism $\mathrm{Tr}$ is defined by

$$
\operatorname{Tr}([E \rightarrow F])=\bigoplus_{g \in G^{T}} \bigoplus_{\zeta \in \mathbb{C}^{*}} \zeta\left[E_{\zeta} \rightarrow F_{\zeta}\right]
$$

Remark 1.1. The above decomposition is proved in [2] in the case of absolute K-theory. However, using the long exact sequence of a pair, it is straightforward to extend the result to relative K-theory as well.

The standard Chern character map gives an isomorphism

$$
\operatorname{ch}: K^{0}\left(\operatorname{Fix}_{g}\left(\mathbb{C}^{3}\right), \operatorname{Fix}_{g}\left(V_{1}^{T}\right)\right) \otimes \mathbb{C} \longrightarrow H^{\mathrm{ev}}\left(\operatorname{Fix}_{g}\left(\mathbb{C}^{3}\right), \operatorname{Fix}_{g}\left(V_{1}^{T}\right) ; \mathbb{C}\right) .
$$

Finally, if $G$ is a finite group acting on a smooth manifold $M$, such that the quotient groupoid $[M / G]$ is an effective orbifold, then $H^{*}(M / G ; \mathbb{C}) \cong\left[H^{*}(M ; \mathbb{C})\right]^{G}$. Indeed, for a finite group $G$ the operation taking $G$-invariants is an exact functor from the category of $G$-vector spaces to the category of vector spaces. Therefore

$$
H^{i}(M / G ; \mathbb{C}) \cong H^{i}\left(\left[\Gamma\left(M, \mathcal{A}_{M}^{*}\right)\right]^{G}\right)=\left[H^{i}\left(M, \mathcal{A}_{M}^{*}\right)\right]^{G} \cong\left[H^{i}(M ; \mathbb{C})\right]^{G},
$$

where $\mathcal{A}_{M}^{*}$ is the sheaf of smooth differential forms on $M$ with complex coefficients, the first isomorphism is Satake's de Rham theorem for orbifolds (see [17]), and the last one is the de Rham's theorem for the manifold $M$. Using the long exact sequence of a pair, we get also that $H^{i}(M / G, N / G ; \mathbb{C}) \cong\left[H^{i}(M, N ; \mathbb{C})\right]^{G}$ for any $G$-invariant submanifold $N \subset M$. On the other hand, by definition,

$$
H_{\text {orb }}^{*}\left(\left[\mathbb{C}^{3} / G^{T}\right],\left[V_{1}^{T} / G^{T}\right] ; k\right)=\bigoplus_{g \in G^{T}} H^{*}\left(\operatorname{Fix}_{g}\left(\mathbb{C}^{3}\right) / G^{T}, \operatorname{Fix}_{g}\left(V_{1}^{T}\right) / G^{T} ; k\right), \quad k=\mathbb{Q}, \mathbb{R}, \mathbb{C} .
$$

Therefore, the composition $\widetilde{c h}:=$ ch $\circ$ Tr defines a ring homomorphism

$$
\widetilde{\mathrm{ch}}: K_{\text {orb }}^{0}\left(\left[\mathbb{C}^{3} / G^{T}\right],\left[V_{1}^{T} / G^{T}\right]\right) \otimes \mathbb{C} \longrightarrow H_{\text {orb }}^{\mathrm{ev}}\left(\left[\mathbb{C}^{3} / G^{T}\right],\left[V_{1}^{T} / G^{T}\right] ; \mathbb{C}\right),
$$

which is the orbifold version of the Chern character map. Clearly ch is an isomorphism over $\mathbb{C}$. 
Remark 1.2. Orbifold cohomology $H_{\text {orb }}^{*}$ has two natural gradings - standard topological degree grading coming from the topological space underlying the orbit space and Chen-Ruan grading. In this paper we work with the topological grading and the topological cup product.

Let us recall also the definition of the $\Gamma$-class. If $E \in K_{\text {orb }}^{0}\left(\left[\mathbb{C}^{3} / G^{T}\right]\right):=K_{G^{T}}^{0}\left(\mathbb{C}^{3}\right)$ is an orbifold vector bundle and $\operatorname{Tr}(E)=\sum_{g} \sum_{\zeta} \zeta E_{\zeta}$, then each eigenvalue $\zeta=e^{2 \pi i \alpha}$, where $0 \leq \alpha<1$ is a rational number and we define

$$
\widehat{\Gamma}(E)=\sum_{g} \prod_{\zeta=e^{2 \pi \mathrm{i} \alpha}} \prod_{i=1}^{\mathrm{rk}\left(E_{\zeta}\right)} \Gamma\left(1-\alpha+\delta_{\zeta, i}\right) \in H_{\mathrm{orb}}^{\mathrm{ev}}\left(\left[\mathbb{C}^{3} / G^{T}\right]\right),
$$

where $\delta_{\zeta, i}\left(1 \leq i \leq \operatorname{rk}\left(E_{\zeta}\right)\right)$ are the Chern roots of the vector bundle $E_{\zeta}$. If $E=\left[T \mathbb{C}^{3} / G^{T}\right]$ is the orbifold tangent bundle, then the $\Gamma$-class is denoted by $\widehat{\Gamma}\left(\left[\mathbb{C}^{3} / G^{T}\right]\right)$. The map (1.2) is defined by the following formula:

$$
\operatorname{ch}_{\Gamma}([E \rightarrow F]):=\frac{1}{2 \pi} \widehat{\Gamma}\left(\left[\mathbb{C}^{3} / G^{T}\right]\right) \cup(2 \pi \mathbf{i})^{\operatorname{deg}_{\mathbb{C}} \iota^{*}} \widetilde{\operatorname{ch}}([E \rightarrow F]),
$$

where $\operatorname{deg}_{\mathbb{C}}(\phi)=i \phi$ for $\phi \in H_{\text {orb }}^{2 i}\left(\left[\mathbb{C}^{3} / G^{T}\right],\left[V_{1}^{T} / G^{T}\right] ; \mathbb{C}\right)$ and $\iota^{*}$ is an involution in orbifold cohomology that exchanges the direct summands corresponding to $g$ and $g^{-1}$. Note that the definition of $\iota^{*}$ makes sense because $\operatorname{Fix}_{g}=$ Fix $_{g^{-1}}$.

Theorem 1.3. There exists a linear isomorphism

$$
\operatorname{mir}: \quad H_{f} \longrightarrow H_{\text {orb }}^{*}\left(\left[\mathbb{C}^{3} / G^{T}\right],\left[V_{1}^{T} / G^{T}\right] ; \mathbb{C}\right),
$$

such that, the map

$$
\operatorname{mir}^{-1} \circ \operatorname{ch}_{\Gamma}: \quad K_{\text {orb }}^{0}\left(\left[\mathbb{C}^{3} / G^{T}\right],\left[V_{1}^{T} / G^{T}\right]\right) \stackrel{\cong}{\longrightarrow} \Psi\left(H_{2}\left(f^{-1}(1) ; \mathbb{Z}\right)\right)
$$

is an isomorphism of Abelian groups.

Unfortunately we do not have a conceptual definition of the map mir. Our definition is on a case by case basis. We expect that $H_{\text {orb }}^{*}\left(\left[\mathbb{C}^{3} / G^{T}\right],\left[V_{1}^{T} / G^{T}\right] ; \mathbb{C}\right)$ has a natural identification with the state space of FJRW-theory under which mir is identified with the mirror map of FanJarvis-Ruan (see [6]). Let us point out also that in all cases the following two properties are satisfied:

1. If $x_{1}^{m_{1}} x_{2}^{m_{2}} x_{3}^{m_{3}}$ is a homogeneous monomial representing a vector in $H_{f}$, then its image under mir is in the twisted sector corresponding to $g=\bar{\rho}_{1}^{m_{1}+1} \bar{\rho}_{2}^{m_{2}+1} \bar{\rho}_{3}^{m_{3}+1}$.

2. The map mir is defined over $\mathbb{Q}$, that is, mir provides an isomorphism

$$
\mathbb{Q}\left[x_{1}, x_{2}, x_{3}\right] /\left(f_{x_{1}}, f_{x_{2}}, f_{x_{3}}\right) \cong H_{\text {orb }}^{*}\left(\left[\mathbb{C}^{3} / G^{T}\right],\left[V_{1}^{T} / G^{T}\right] ; \mathbb{Q}\right) .
$$

\section{Period map image of the Milnor lattice}

\subsection{Suspension isomorphism in vanishing homology}

We will reduce the problem of computing periods of the hypersurface $V_{\lambda}$ to computing periods of the Riemann surfaces

$$
M_{\mu}:=\left\{\left(x_{1}, x_{2}\right) \in \mathbb{C}^{2} \mid g\left(x_{1}, x_{2}\right)=\mu\right\} .
$$


Consider the map $V_{\lambda} \rightarrow \mathbb{C},\left(x_{1}, x_{2}, x_{3}\right) \mapsto g\left(x_{1}, x_{2}\right)$. The fibers of this map are given by

$$
V_{\lambda, \mu}:=M_{\mu} \times\{-\sqrt{\lambda-\mu}, \sqrt{\lambda-\mu}\} .
$$

Suppose now that $A \in H_{1}\left(M_{\lambda} ; \mathbb{Z}\right)$ is any cycle. The following two maps

$$
\phi_{ \pm}: A \times[0,1] \rightarrow V_{\lambda}, \quad\left(x_{1}, x_{2}, t\right) \mapsto\left(t^{c_{1}} x_{1}, t^{c_{2}} x_{2}, \pm \sqrt{\lambda(1-t)}\right)
$$

have images that fit together and give a two-dimensional cycle $\alpha \in V_{\lambda}$, that is, $\alpha=\Sigma A$ is the suspension of the cycle $A$. It is known that the above suspension operation $\Sigma: H_{1}\left(M_{\lambda} ; \mathbb{Z}\right) \rightarrow$ $H_{2}\left(V_{\lambda} ; \mathbb{Z}\right)$ is an isomorphism (see $[1$, Theorem 2.9]).

Note that we may choose the basis of $H_{f}$ to be such that $\phi_{i}=\phi_{i}\left(x_{1}, x_{2}\right)$ does not depend on $x_{3}$. Then the integral

$$
\frac{1}{2 \pi} \int_{\alpha_{\lambda}} \phi_{i} \frac{\omega}{d f}=\frac{1}{2 \pi} \partial_{\lambda} \int_{\alpha_{\lambda}} d^{-1}\left(\phi_{i} \omega\right)=\frac{1}{2 \pi} \partial_{\lambda} \int_{\alpha_{\lambda}} x_{3} \phi_{i}\left(x_{1}, x_{2}\right) d x_{1} \wedge d x_{2},
$$

where in the first equality we used the Stoke's theorem (see [1, Lemma 7.2]). Using Fubini's theorem (see [1, Lemma 7.2]), we have

$$
\begin{aligned}
\int_{\alpha_{\lambda}} x_{3} \phi_{i}\left(x_{1}, x_{2}\right) d x_{1} \wedge d x_{2}= & \int_{0}^{\lambda}(\lambda-\mu)^{1 / 2} \int_{A_{\mu}} \frac{\phi_{i}\left(x_{1}, x_{2}\right) d x_{1} d x_{2}}{d g} d \mu \\
& -\int_{\lambda}^{0}\left(-(\lambda-\mu)^{1 / 2}\right) \int_{A_{\mu}} \frac{\phi_{i}\left(x_{1}, x_{2}\right) d x_{1} d x_{2}}{d g} d \mu
\end{aligned}
$$

where the first integral represents integrating over $\phi_{+}(A \times[0,1])$, the second one over $\phi_{-}(A \times$ $[0,1])$, and $A_{\mu} \in H_{1}\left(M_{\mu}\right)$ for $\mu=\lambda t$ is obtained from $A$ via the rescaling $\left(x_{1}, x_{2}\right) \mapsto\left(t^{c_{1}} x_{1}, t^{c_{2}} x_{2}\right)$. We get

$$
\frac{1}{2 \pi} \int_{\alpha_{\lambda}} \phi_{i} \frac{\omega}{d f}=\frac{1}{\pi} \partial_{\lambda} \int_{0}^{\lambda}(\lambda-\mu)^{1 / 2} \int_{A_{\mu}} \frac{\phi_{i}\left(x_{1}, x_{2}\right) d x_{1} d x_{2}}{d g} d \mu .
$$

The image of the Milnor lattice $H_{2}\left(V_{1} ; \mathbb{Z}\right)$ will be computed with formula (2.1).

\subsection{Simple singularities and root systems}

Let us first recall several well known facts about simple singularities, which will be needed in our computation (see [1, Theorem 3.14]). The analytic continuation of $I_{\alpha}^{(-1)}(\lambda)$ along a loop around $\lambda=0$ yields $I_{\sigma(\alpha)}^{(-1)}(\lambda)$, where $\sigma: H_{2}\left(V_{1} ; \mathbb{Z}\right) \rightarrow H_{2}\left(V_{1} ; \mathbb{Z}\right)$ is the so-called classical monodromy operator. Recalling the definition of $\Psi$ (see formula (1.1)), we get the following relation:

$$
\Psi(\sigma(\alpha))=-e^{2 \pi \mathbf{i} \theta} \Psi(\alpha),
$$

where $\mathbf{i}:=\sqrt{-1}$. In particular, knowing the image of one cycle $\alpha$ allows us to find the image of the entire $\sigma$-orbit of $\alpha$.

Let us define

$$
(\alpha \mid \beta):=\lambda\left(I_{\alpha}^{(0)}(\lambda), I_{\beta}^{(0)}(\lambda)\right),
$$

where $I_{\alpha}^{(0)}(\lambda):=\partial_{\lambda} I_{\alpha}^{(-1)}(\lambda)$. It is straightforward to check that

$$
(\alpha \mid \beta)=\frac{1}{\pi}(\Psi(\alpha), \cos (\pi \theta) \Psi(\beta)) .
$$

It is known that $(\alpha \mid \beta)=-\alpha \circ \beta$, where $\circ$ is the intersection pairing (see $[10,16])$. In particular, the form $(\mid)$ takes integer values on the Milnor lattice.

Finally, let us also recall that we have the following remarkable facts (see [1, Theorem 3.14]): 
1. The set of vanishing cycles of the singularity $f$ coincides with the set of all $\alpha \in H_{2}\left(V_{1} ; \mathbb{Z}\right)$ such that $(\alpha \mid \alpha)=2$.

2. The triple (Milnor lattice, set of vanishing cycles, pairing $(\mid)$ ) form a root system of the same type as the type of the singularity $f$, that is, the set of vanishing cycles corresponds to the roots, the Milnor lattice corresponds to the root lattice, and $(\mid)$ corresponds to the invariant bilinear form.

3. The classical monodromy corresponds to a Coxeter transformation.

\section{$2.3 \quad A_{N}$-singularity}

Let us fix the following basis of $H_{f}$ :

$$
\phi_{i}=x_{1}^{i-1}, \quad 1 \leqslant i \leqslant N .
$$

The residue pairing takes the form

$$
\left(\phi_{i}, \phi_{j}\right)=\frac{1}{4 h} \delta_{i+j, h}, \quad 1 \leqslant i, j \leqslant N,
$$

where $h=N+1$ is the Coxeter number. The Riemann surface $M_{\mu}$ for $\mu \neq 0$ is a non-singular curve in $\mathbb{C}^{2}$ defined by the equation $x_{1}^{N+1}+x_{2}^{2}=\mu$. The projection $\left(x_{1}, x_{2}\right) \mapsto x_{1}$ defines a degree 2 branched covering $M_{\mu} \rightarrow \mathbb{C}$, with branching points $x_{1, k}=\mu^{\frac{1}{N+1}} \eta_{N+1}^{k}\left(k \in \mathbb{Z}_{N+1}\right)$, where $\eta_{N+1}:=e^{2 \pi \mathbf{i} /(N+1)}$ and $\mathbb{Z}_{N+1}:=\mathbb{Z} /(N+1) \mathbb{Z}$.

Let us construct a basis of $H_{1}\left(M_{\mu} ; \mathbb{Z}\right) \cong \mathbb{Z}^{N}$. Cycles on $M_{\mu}$ can be visualized easily via their projections on the $x_{1}$-plane $\mathbb{C}$. Let $L_{k}\left(k \in \mathbb{Z}_{N+1}\right)$ be the line segment $\left[0, x_{1, k}\right]$ (in the $x_{1}$-plane). Let $A_{k}^{\prime}$ be a loop in the $x_{1}$-plane that starts at $x_{1}=0$, it goes along the line segment $L_{k}$, just before hitting the branched point $x_{1, k}$ it makes a small loop $C_{k}$ counterclockwise around $x_{1, k}$, it returns back to the starting point along the line segment $L_{k}$, and then the loop continues to travel in a similar fashion along $L_{k+1}$ except that this time we make a small loop $C_{k+1}^{-1}$ clockwise around $x_{1, k+1}$. In other words $A_{k}^{\prime}=L_{k+1}^{-1} \circ C_{k+1}^{-1} \circ L_{k+1} \circ L_{k}^{-1} \circ C_{k} \circ L_{k}$. Note that $A_{k}^{\prime}$ lifts to two loops $A_{k, a}, a \in \mathbb{Z}_{2}$ on $M_{\mu}$, where the starting point of $A_{k}^{\prime}$ lifts to $x_{2, k, a}:=\mu^{\frac{1}{2}}(-1)^{a}$. The cycles $A_{k, a}$ satisfy the following relations $A_{k, 0}=-A_{k, 1}$ and $\sum_{k=0}^{N} A_{k, a}=0$. Let us asume $a \in \mathbb{Z}_{2} \backslash\{0\}$ and $k \in \mathbb{Z}_{N+1} \backslash\{0\}$, then we get $N$ loops whose homology classes, as we will see later on, represent a basis of $H_{1}\left(M_{\mu} ; \mathbb{Z}\right)$.

Let us compute the periods of the holomorphic forms

$$
\phi_{i}\left(x_{1}, x_{2}\right) \frac{d x_{1} d x_{2}}{d g}=-\frac{x_{1}^{i-1} d x_{1}}{2 x_{2}}
$$

along the cycles $A_{k, a}$. The paths $L_{k}$ and $C_{k}$ can be parametrized as follows:

$$
\begin{array}{ll}
L_{k}: x_{1}=\eta_{N+1}^{k} \mu^{\frac{1}{N+1}} t^{\frac{1}{N+1}}, \quad 0 \leqslant t \leqslant\left(1-\frac{\epsilon}{\mu^{\frac{1}{N+1}}}\right)^{N+1}, \\
C_{k}: x_{1}=\eta_{N+1}^{k} \mu^{\frac{1}{N+1}}+\epsilon e^{\mathbf{i} \theta}, \quad \frac{2 k-N-1}{N+1} \pi \leqslant \theta \leqslant \frac{2 k+N+1}{N+1} \pi .
\end{array}
$$

The integrals along the lifts of $C_{k}$ contribute to the period integral terms of order $O\left(\epsilon^{\frac{1}{2}}\right)$. These terms vanish in the limit $\epsilon \rightarrow 0$. The periods that we want to compute are independent of $\epsilon$ for homotopy reasons. Therefore, by passing to the limit $\epsilon \rightarrow 0$ we get

$$
\int_{A_{k, a}} \phi_{i}\left(x_{1}, x_{2}\right) \frac{d x_{1} d x_{2}}{d g}=(1-(-1))\left(\int_{L_{k, a}}-\int_{L_{k+1, a}}\right) \frac{-x_{1}^{i-1} d x_{1}}{2 x_{2}}
$$




$$
=\left(\int_{L_{k+1, a}}-\int_{L_{k, a}}\right) \frac{x_{1}^{i-1} d x_{1}}{x_{2}}
$$

The integrals along $L_{k, a}$ can be expressed in terms of Euler's Beta function $B(a, b):=\frac{\Gamma(a) \Gamma(b)}{\Gamma(a+b)}$,

$$
\int_{L_{k, a}} \frac{x_{1}^{i-1} d x_{1}}{x_{2}}=(-1)^{a} \int_{0}^{1} \frac{\eta_{N+1}^{k i} \mu^{\frac{i}{N+1}} t^{\frac{i}{N+1}}-1}{(N+1) \mu^{\frac{1}{2}}(1-t)^{\frac{1}{2}}}=(-1)^{a} \frac{\eta_{N+1}^{k i} \mu^{\frac{i}{N+1}-\frac{1}{2}}}{N+1} B\left(\frac{i}{N+1}, \frac{1}{2}\right) .
$$

Let $\alpha_{k, a}=\Sigma A_{k, a}$ be the suspension. Recalling formula (2.1) and using that

$$
\int_{0}^{\lambda}(\lambda-\mu)^{1 / 2} \mu^{a} d \mu=\lambda^{a+3 / 2} B(a+1,3 / 2),
$$

we get

$$
\begin{aligned}
\left(I_{\alpha_{k, a}}^{(-1)}(\lambda), \phi_{i}\right) & :=\frac{1}{2 \pi} \int_{\alpha_{k, a}} \phi_{i} \frac{\omega}{d f}=\frac{1}{\pi} \partial_{\lambda} \int_{0}^{\lambda}(\lambda-\mu)^{\frac{1}{2}} \int_{A_{k, a}} \phi_{i}\left(x_{1}, x_{2}\right) \frac{d x_{1} d x_{2}}{d g} d \mu \\
& =(-1)^{a} \frac{\eta_{N+1}^{k i}\left(\eta_{N+1}^{i}-1\right)}{2 i} \lambda^{\frac{i}{N+1}} .
\end{aligned}
$$

Recalling the formulas for the residue pairing we get

$$
I_{\alpha_{k, a}}^{(-1)}(\lambda)=4 h \sum_{i=1}^{N}\left(I_{\alpha_{k, a}}^{(-1)}(\lambda), \phi_{h-i}\right) \phi_{i}
$$

By definition $\theta\left(\phi_{i}\right)=\left(\frac{1}{2}-\frac{i}{N+1}\right) \phi_{i}$. Therefore, using (1.1), we get

$$
\Psi\left(\alpha_{k, a}\right)=(-1)^{a} 2 \sum_{i=1}^{N} \eta_{N+1}^{-k i}\left(\eta_{N+1}^{-i}-1\right) \Gamma\left(1-\frac{i}{N+1}\right) \phi_{i} .
$$

Let us point out that formula (2.2) yields the following formulas for the classical monodromy operator

$$
\sigma\left(\alpha_{k, a}\right)=-\alpha_{k+1, a+1}=\alpha_{k+1, a}, \quad k \in \mathbb{Z}_{N+1}, \quad a \in \mathbb{Z}_{2} .
$$

The intersection pairing takes the form

$$
\begin{aligned}
\left(\alpha_{k, 1} \mid \alpha_{l, 1}\right) & =\frac{1}{\pi}\left(\Psi\left(\alpha_{k, 1}\right), \cos (\pi \theta) \Psi\left(\alpha_{l, 1}\right)\right) \\
& =\frac{2}{h} \sum_{i=1}^{N} \eta_{N+1}^{(l-k) i}\left(1-\cos \left(\frac{2 i \pi}{h}\right)\right)=2 \delta_{k, l}-\delta_{l-k, 1}-\delta_{l-k, N}
\end{aligned}
$$

where $k, l \in \mathbb{Z}_{N+1}$ and the Kronecker delta is also on $\mathbb{Z}_{N+1}$. Note that $\left(\alpha_{k, 1} \mid \alpha_{k, 1}\right)=2$, so $\alpha_{k, 1}$ is a vanishing cycle. The determinant of the intersection pairing in the basis $\left\{\alpha_{k, 1}\right\}$, that is, the determinant of the matrix $\left(\alpha_{k, 1} \mid \alpha_{l, 1}\right)_{k, l=1}^{N}$ is $N+1$, which coincides with the determinant of the Cartan matrix of the simple Lie algebra of type $A_{N}$. Therefore, $\left\{\alpha_{k, 1}\right\}$ is a $\mathbb{Z}$-basis of the root lattice, that is, $H_{2}\left(V_{\lambda} ; \mathbb{Z}\right)$ and hence their images $\Psi\left(\alpha_{k, 1}\right)$ (see formula $\left.(2.5)\right)$ give a basis for the image of the Milnor lattice in $H_{f}$. 


\section{$2.4 D_{N^{-S i n g u l a r i t y}}$}

Let us fix the following basis of $H_{f}$ :

$$
\phi_{i}\left(x_{1}, x_{2}\right)= \begin{cases}x_{2}^{i-1} & \text { if } 1 \leq i \leq N-1, \\ 2 x_{1} & \text { if } i=N .\end{cases}
$$

The residue pairing takes the form

$$
\left(\phi_{i}, \phi_{j}\right)=\frac{1}{2 h} \delta_{i+j, N}, \quad 1 \leq i, j \leq N-1, \quad\left(\phi_{i}, \phi_{N}\right)=-\delta_{i, N}, \quad 1 \leq i \leq N,
$$

where $h=2 N-2$ is the Coxeter number. The Riemann surface $M_{\mu}$ for $\mu \neq 0$ is a non-singular curve in $\mathbb{C}^{2}$ defined by the equation $x_{1}^{2} x_{2}+x_{2}^{N-1}=\mu$. The projection $\left(x_{1}, x_{2}\right) \mapsto x_{2}$ defines a degree 2 branched covering $M_{\mu} \rightarrow \mathbb{C}^{*}$, with branching points $x_{2, k}=\mu^{\frac{1}{N-1}} \eta^{2 k}(1 \leq k \leq N-1)$, where $\eta=e^{2 \pi \mathbf{i} / h}$. Let $A_{k}^{\prime}$ be a simple loop in $\mathbb{C}^{*}$ around the line segment $L_{k}:=\left[0, x_{2, k}\right]$, that is, $A_{k}^{\prime}$ is a loop starting at a point on the line segment $L_{k}$ sufficiently close to 0 , it goes along the line segment $L_{k}$, just before hitting the branch point $x_{2, k}$ it makes a small loop $C_{k}$ around it and it returns back to the starting point along the line segment $L_{k}$, and finally it makes a small loop $C_{0}$ around 0. Clearly, the loop $A_{k}^{\prime}=C_{0} \circ L_{k}^{-1} \circ C_{k} \circ L_{k}$ lifts to a loop $A_{k}$ in $M_{\mu}$. Let us compute the periods of the holomorphic forms

$$
\phi_{i}\left(x_{1}, x_{2}\right) \frac{d x_{1} d x_{2}}{d g}= \begin{cases}\frac{x_{2}^{i-1} d x_{2}}{2 x_{1} x_{2}} & \text { if } 1 \leq i \leq N-1, \\ \frac{d x_{2}}{x_{2}} & \text { if } i=N,\end{cases}
$$

along the cycle $A_{k}$. If $i=N$, then the period integral is just $2 \pi \mathbf{i}$. Suppose that $1 \leq i \leq N-1$. Let us parametrize $A_{k}^{\prime}$ as follows:

$$
\begin{aligned}
& C_{0}: x_{2}=\epsilon e^{\mathbf{i} \theta}, \quad 0 \leq \theta \leq 2 \pi, \\
& L_{k}: x_{2}=\mu^{\frac{1}{N-1}} \eta^{2 k} t, \quad \epsilon \mu^{-\frac{1}{N-1}} \leq t \leq 1-\epsilon \mu^{-\frac{1}{N-1}}, \\
& C_{k}: x_{2}=\mu^{\frac{1}{N-1}} \eta^{2 k}+\epsilon e^{\mathbf{i} \theta}, \quad 0 \leq \theta \leq 2 \pi .
\end{aligned}
$$

The integrals along the lifts of $C_{0}$ and $C_{k}$ contribute to the period integral terms of orders respectively $O\left(\epsilon^{i-1 / 2}\right)$ and $O\left(\epsilon^{1 / 2}\right)$. These terms vanish in the limit $\epsilon \rightarrow 0$. The two lifts of $L_{k}$, before and after going around the branch point $x_{2, k}$, have parametrizations, such that,

$$
x_{2}=\mu^{\frac{1}{N-1}} \eta^{2 k} t, \quad x_{1} x_{2}=\sqrt{\left(\mu-x_{2}^{N-1}\right) x_{2}}=\eta^{k} \mu^{1 / 2+1 / h}\left(1-t^{N-1}\right)^{1 / 2} t^{1 / 2},
$$

where $t$ varies from 0 to 1 , and

$$
x_{2}=\mu^{\frac{1}{N-1}} \eta^{2 k} t, \quad x_{1} x_{2}=-\sqrt{\left(\mu-x_{2}^{N-1}\right) x_{2}}=-\eta^{k} \mu^{1 / 2+1 / h}\left(1-t^{N-1}\right)^{1 / 2} t^{1 / 2},
$$

where $t$ varies from 1 to 0 . Now it is clear that the period integral, after passing to the limit $\epsilon \rightarrow 0$, takes the form

$$
\int_{A_{k}} \phi_{i} \frac{d x_{1} d x_{2}}{d g}=\mu^{\frac{m_{i}}{h}-\frac{1}{2}} \eta^{m_{i} k} \int_{0}^{1} t^{i-\frac{3}{2}}\left(1-t^{N-1}\right)^{-1 / 2} d t
$$

where $m_{i}:=2 i-1(1 \leq i \leq N-1)$. The above integral can be computed as follows,

$$
\int_{0}^{1} t^{i-3 / 2}\left(1-t^{N-1}\right)^{-1 / 2} d t=\frac{1}{N-1} \int_{0}^{1} s^{(2 i-1) \frac{1}{h}-1}(1-s)^{-\frac{1}{2}} d s=\frac{1}{N-1} B\left(\frac{m_{i}}{h}, \frac{1}{2}\right) .
$$


We get the following formulas:

$$
\int_{A_{k}} \phi_{i} \frac{d x_{1} d x_{2}}{d g}= \begin{cases}\frac{1}{N-1} \mu^{\frac{m_{i}}{h}-\frac{1}{2}} \eta^{m_{i} k} B\left(\frac{m_{i}}{h}, \frac{1}{2}\right), & \text { if } 1 \leq i \leq N-1, \\ 2 \pi \mathbf{i}, & \text { if } i=N .\end{cases}
$$

Let $\alpha_{k}=\Sigma A_{k}$ be the suspension. Recalling formula (2.1) and using (2.4), we get

$$
\frac{1}{2 \pi} \int_{\alpha_{k}} \phi_{i} \frac{\omega}{d f}= \begin{cases}\frac{1}{h} \eta^{m_{i} k} \frac{\lambda^{m_{i} / h}}{m_{i} / h}, & \text { if } 1 \leq i \leq N-1 \\ 2 \mathbf{i} \lambda^{1 / 2}, & \text { if } i=N\end{cases}
$$

Therefore,

$$
I_{\alpha_{k}}^{(-1)}(\lambda)=2 \sum_{i=1}^{N-1} \eta^{m_{i} k} \frac{\lambda^{m_{i} / h}}{m_{i} / h} \phi_{N-i}-2 \mathbf{i} \lambda^{1 / 2} \phi_{N} .
$$

Note that $\theta\left(\phi_{i}\right)=\left(\frac{m_{N-i}}{h}-\frac{1}{2}\right) \phi_{i}$ for $1 \leq i \leq N-1$ and $\theta\left(\phi_{N}\right)=0$. Therefore

$$
\Psi\left(\alpha_{k}\right)=2 \sum_{i=1}^{N-1} \eta^{m_{i} k} \Gamma\left(m_{i} / h\right) \phi_{N-i}-\mathbf{i} \Gamma\left(m_{N} / h\right) \phi_{N},
$$

where $m_{N}=N-1$.

Remark 2.1. The numbers $\frac{m_{i}}{h}=\frac{2 i-1}{h}(1 \leq i \leq N-1), \frac{m_{N}}{h}=\frac{1}{2}$ are the Coxeter exponents.

Put

$$
v_{k}=2 \sum_{i=1}^{N-1} \eta^{m_{i} k} \Gamma\left(m_{i} / h\right) \phi_{N-i}, \quad 1 \leq k \leq N-1,
$$

and $v_{N}=\mathbf{i} \Gamma\left(m_{N} / h\right) \phi_{N}$.

Proposition 2.2. The image of the Milnor lattice under the map $\Psi$ is the lattice in $H_{f}$ with $\mathbb{Z}$-basis

$$
\beta_{1}=v_{1}-v_{2}, \ldots, \beta_{N-1}=v_{N-1}-v_{N}, \quad \beta_{N}=v_{N-1}+v_{N} .
$$

Proof. Using formula (2.3), it is straightforward to check that $\left\{v_{i}\right\}_{1 \leq i \leq N}$ is an orthonormal basis of $H_{f}$ with respect to the intersection pairing, that is, $\left(v_{i} \mid v_{j}\right)=\delta_{i, j}$. We have $\Psi\left(\alpha_{k}\right)=v_{k}-v_{N}$ and $\Psi\left(\sigma \alpha_{k}\right)-\Psi\left(\alpha_{k+1}\right)=2 v_{N}$. Therefore, $\beta_{i}$ belongs to the image of the Milnor lattice. On the other hand, since $\left(\beta_{i} \mid \beta_{i}\right)=2$, we get that $\beta_{i}$ is the image of a vanishing cycle. Recalling the root system interpretation of the set of vanishing cycles, we get that $\beta_{i}(1 \leq i \leq N)$ are simple roots and that the corresponding Dynkin diagram is the Dynkin diagram of type $D_{N}$. Since the Milnor lattice is spanned by the set of vanishing cycles, the claim of the proposition follows.

\section{$2.5 \quad E_{6}$-singularity}

Let us fix the following basis of $H_{f}$ :

$$
\phi_{i}= \begin{cases}x_{2}^{i-1} & \text { if } 1 \leqslant i \leqslant 3 \\ x_{1} x_{2}^{i-4} & \text { if } 4 \leqslant i \leqslant 6\end{cases}
$$


The residue pairing takes the form

$$
\left(\phi_{i}, \phi_{j}\right)=\frac{1}{2 h} \delta_{i+j, 7}, \quad 1 \leqslant i, j \leqslant 6,
$$

where $h=12$ is the Coxeter number. The Riemann surface $M_{\mu}$ for $\mu \neq 0$ is a non-singular curve in $\mathbb{C}^{2}$ defined by the equation $x_{1}^{3}+x_{2}^{4}=\mu$. The projection $\left(x_{1}, x_{2}\right) \mapsto x_{2}$ defines a degree 3 branched covering $M_{\mu} \rightarrow \mathbb{C}$, with branching points $x_{2, k}=\mu^{\frac{1}{4}} \mathbf{i}^{k}, k \in \mathbb{Z}_{4}$.

Let $L_{k}\left(k \in \mathbb{Z}_{4}\right)$ be the line segment $\left[0, \mu^{\frac{1}{4}} \mathbf{i}^{k}\right]$. Let $A_{k}^{\prime}$ be a loop in the $x_{2}$-plane $\mathbb{C}$ going around the branch points $x_{2, k}$ and $x_{2, k+1}$ in the following way: the loop starts at 0 , it goes along the line segment $L_{k}$, just before hitting the branch point $x_{2, k}$ it makes a small loop $C_{k}$ counterclockwise around $x_{2, k}$, it returns back to the starting point along $L_{k}$; then the loop travels in a similar fashion along $L_{k+1}$ except that this time we make a small loop $C_{k+1}^{-1}$ in clockwise direction around $x_{2, k+1}$. Clearly, the loop $A_{k}^{\prime}=L_{k+1}^{-1} \circ C_{k+1}^{-1} \circ L_{k+1} \circ L_{k}^{-1} \circ C_{k} \circ L_{k}$ lifts to three loops $A_{k, a}, a \in \mathbb{Z}_{3}$ in $M_{\mu}$, depending on how we choose the lift of the base point, i.e., the $x_{1}$-coordinate of the lift of the base point of $A_{k}^{\prime}$ could take the following values: $x_{1, a}=\mu^{\frac{1}{3}} \eta_{3}^{a}$, where $\eta_{3}:=e^{\frac{2 \pi \mathbf{i}}{3}}$.

Let us consider the loops $A_{k, a}$ with $a \in \mathbb{Z}_{3} \backslash\{0\}$ and $k \in \mathbb{Z}_{4} \backslash\{0\}$. Let us compute the periods of the holomorphic forms

$$
\phi_{i}\left(x_{1}, x_{2}\right) \frac{d x_{1} d x_{2}}{d g}= \begin{cases}\frac{x_{2}^{i-1} d x_{2}}{3 x_{1}^{2}} & \text { if } 1 \leqslant i \leqslant 3, \\ \frac{x_{2}^{i-4} d x_{2}}{3 x_{1}} & \text { if } 4 \leqslant i \leqslant 6,\end{cases}
$$

along the cycles $A_{k, a}$. As a byproduct of our computation we will get that the homology classes of these 6 loops form a basis of $H_{1}\left(M_{\mu} ; \mathbb{Z}\right)$. Let us parametrize $A_{k}^{\prime}$ as follows:

$$
\begin{aligned}
& L_{k}: x_{2}=\mathbf{i}^{k} \mu^{\frac{1}{4}} t^{\frac{1}{4}}, \quad 0 \leqslant t \leqslant\left(1-\frac{\epsilon}{\mu^{\frac{1}{4}}}\right)^{4}, \\
& C_{k}: x_{2}=\mathbf{i}^{k} \mu^{\frac{1}{4}}+\epsilon e^{\mathbf{i} \theta}, \quad \frac{k-2}{2} \pi \leqslant \theta \leqslant \frac{k+2}{2} \pi .
\end{aligned}
$$

The integrals along the lifts of $C_{k}$ contribute to the period integral terms of orders

$$
\begin{cases}O\left(\epsilon^{\frac{1}{3}}\right) & \text { if } 1 \leqslant i \leqslant 3 \\ O\left(\epsilon^{\frac{2}{3}}\right) & \text { if } 4 \leqslant i \leqslant 6 .\end{cases}
$$

These terms vanish in the limit $\epsilon \rightarrow 0$. Therefore, under this limit, the periods of the holomorphic forms

$$
\int_{A_{k, a}} \phi_{i}\left(x_{1}, x_{2}\right) \frac{d x_{1} d x_{2}}{d g}=\left\{\begin{array}{l}
\left(1-\eta_{3}^{-2}\right)\left(\int_{L_{k, a}}-\int_{L_{k+1, a}}\right) \frac{\phi_{i} d x_{2}}{3 x_{1}^{2}} \quad \text { if } 1 \leqslant i \leqslant 3, \\
\left(1-\eta_{3}^{-1}\right)\left(\int_{L_{k, a}}-\int_{L_{k+1, a}}\right) \frac{\phi_{i} d x_{2}}{3 x_{1}^{2}} \quad \text { if } 4 \leqslant i \leqslant 6,
\end{array}\right.
$$

where $\eta_{3}:=e^{\frac{2 \pi \mathbf{i}}{3}}$ and the integral

$$
\int_{L_{k, a}} \frac{\phi_{i} d x_{2}}{3 x_{1}^{2}}= \begin{cases}\int_{0}^{1} \frac{\mathbf{i}^{k i} \mu^{\frac{i}{4}} t^{\frac{i}{4}-1} d t}{12 \mu^{\frac{2}{3}}(1-t)^{\frac{2}{3}} \eta_{3}^{2 a}}=\frac{\mathbf{i}^{k i}}{12} \eta_{3}^{a} \mu^{\frac{i}{4}-\frac{2}{3}} B\left(\frac{i}{4}, \frac{1}{3}\right) & \text { if } 1 \leqslant i \leqslant 3, \\ \int_{0}^{1} \frac{\mathbf{i}^{k(i-3)} \mu^{\frac{i-3}{4}} t^{\frac{i-3}{4}}-1}{12 \mu^{\frac{1}{3}}(1-t)^{\frac{1}{3}} \eta_{3}^{a}}=\frac{\mathbf{i}^{k(i-3)}}{12} \eta_{3}^{2 a} \mu^{\frac{i-3}{4}-\frac{1}{3}} B\left(\frac{i-3}{4}, \frac{2}{3}\right) & \text { if } 4 \leqslant i \leqslant 6 .\end{cases}
$$


Then,

$$
\begin{aligned}
\int_{A_{k, a}} \phi_{i}\left(x_{1}, x_{2}\right) \frac{d x_{1} d x_{2}}{d g} & \text { if } 1 \leqslant i \leqslant 3, \\
= & \begin{array}{ll}
\left(1-\eta_{3}^{-2}\right)\left(1-\mathbf{i}^{i}\right) \frac{\mathbf{i}^{k i}}{12} \eta_{3}^{a} \mu^{\frac{i}{4}-\frac{2}{3}} B\left(\frac{i}{4}, \frac{1}{3}\right) & \text { if } 4 \leqslant i \leqslant 6 . \\
\left(1-\eta_{3}^{-1}\right)\left(1-\mathbf{i}^{i-3}\right) \frac{\mathbf{i}^{k(i-3)}}{12} \eta_{3}^{2 a} \mu^{\frac{i-3}{4}-\frac{1}{3}} B\left(\frac{i-3}{4}, \frac{2}{3}\right)
\end{array}
\end{aligned}
$$

Let $\alpha_{k, a}=\Sigma A_{k, a}$ be the suspension. Recalling formula (2.1) and using (2.4)

$$
\begin{aligned}
\left(I_{\alpha_{k, a}}^{(-1)}(\lambda), \phi_{i}\right) & :=\frac{1}{2 \pi} \int_{\alpha_{k, a}} \phi_{i} \frac{\omega}{d f}=\frac{1}{\pi} \partial_{\lambda} \int_{0}^{\lambda}(\lambda-\mu)^{\frac{1}{2}} \int_{A_{k, a}} \phi_{i}\left(x_{1}, x_{2}\right) \frac{d x_{1} d x_{2}}{d g} d \mu \\
& = \begin{cases}\frac{\sqrt{3}}{12 \pi} \eta_{3}^{a} e^{-\frac{\pi}{6} \mathbf{i}} \lambda^{\frac{i}{4}-\frac{1}{6}} \frac{\Gamma\left(\frac{3}{2}\right) \Gamma\left(\frac{i}{4}\right) \Gamma\left(\frac{1}{3}\right)}{\Gamma\left(\frac{i}{4}+\frac{5}{6}\right)} \mathbf{i}^{k i}\left(1-\mathbf{i}^{i}\right) & \text { if } 1 \leqslant i \leqslant 3, \\
\frac{\sqrt{3}}{12 \pi} \eta_{3}^{2 a} e^{\frac{\pi}{6}} \mathbf{i} \lambda^{\frac{i-3}{4}+\frac{1}{6}} \frac{\Gamma\left(\frac{3}{2}\right) \Gamma\left(\frac{i-3}{4}\right) \Gamma\left(\frac{2}{3}\right)}{\Gamma\left(\frac{i-3}{4}+\frac{7}{6}\right)} \mathbf{i}^{k(i-3)}\left(1-\mathbf{i}^{i-3}\right) & \text { if } 4 \leqslant i \leqslant 6 .\end{cases}
\end{aligned}
$$

Recalling the formulas for the residue pairing in the basis $\left\{\phi_{i}\right\}$ we get

$$
I_{\alpha_{k, a}}^{(-1)}(\lambda)=2 h \sum_{i=1}^{6}\left(I_{\alpha_{k, a}}^{(-1)}(\lambda), \phi_{7-i}\right) \phi_{i} .
$$

Recalling formula (1.1) and using that by definition

$$
\theta\left(\phi_{i}\right)= \begin{cases}\left(\frac{2}{3}-\frac{i}{4}\right) \phi_{i} & \text { if } 1 \leqslant i \leqslant 3 \\ \left(\frac{1}{3}-\frac{i-3}{4}\right) \phi_{i} & \text { if } 4 \leqslant i \leqslant 6\end{cases}
$$

we get

$$
\begin{aligned}
\Psi\left(\alpha_{k, a}\right)= & \sqrt{\frac{3}{\pi}} \sum_{i=1}^{3} e^{\frac{\pi}{6}} \mathbf{i} \eta_{3}^{2 a} \Gamma\left(1-\frac{i}{4}\right) \Gamma\left(\frac{2}{3}\right) \mathbf{i}^{-k i}\left(1-\mathbf{i}^{-i}\right) \phi_{i} \\
& +\sqrt{\frac{3}{\pi}} \sum_{i=4}^{6} e^{-\frac{\pi}{6} \mathbf{i}} \eta_{3}^{a} \Gamma\left(1-\frac{i-3}{4}\right) \Gamma\left(\frac{1}{3}\right) \mathbf{i}^{k(3-i)}\left(1-\mathbf{i}^{3-i}\right) \phi_{i} .
\end{aligned}
$$

Let us also point out that by using formula (2.2), we get the following formulas for the classical monodromy operator:

$$
\sigma\left(\alpha_{k, a}\right)=-\alpha_{k+1, a+1}, \quad k \in \mathbb{Z}_{4}, \quad a \in \mathbb{Z}_{3} .
$$

Recalling formula (2.3), we get that the intersection pairing

$$
\begin{aligned}
\left(\alpha_{k, a} \mid \alpha_{l, b}\right) & =\frac{1}{\pi}\left(\Psi\left(\alpha_{k, a}\right), \cos (\pi \theta) \Psi\left(\alpha_{l, b}\right)\right) \\
& =\frac{1}{8} \sum_{i=1}^{3}\left(\eta_{3}^{b-a} \mathbf{i}^{(l-k) i}+\eta_{3}^{a-b} \mathbf{i}^{(k-l) i}\right) \frac{\cos \left(\left(\frac{i}{4}-\frac{2}{3}\right) \pi\right)}{\sin \left(\frac{i}{4} \pi\right) \sin \left(\frac{\pi}{3}\right)}\left(2-\mathbf{i}^{-i}-\mathbf{i}^{i}\right) \\
& =\sum_{i=1}^{3} \cos \left(\frac{2}{3}(b-a) \pi+\frac{i}{2}(l-k) \pi\right) \frac{\cos \left(\left(\frac{i}{4}-\frac{2}{3}\right) \pi\right)}{\sin \left(\frac{\pi}{3}\right)} \sin \left(\frac{i}{4} \pi\right)
\end{aligned}
$$


Let us identify $\mathbb{Z}_{3} \backslash\{0\}=\{1,2\}$ and $\mathbb{Z}_{4} \backslash\{0\}=\{1,2,3\}$. Every $1 \leq a^{\prime} \leq 6$ can be written uniquely in the form $a^{\prime}=3(a-1)+k$, where $1 \leq a \leq 2$ and $1 \leq k \leq 3$. Let us define $\alpha_{a^{\prime}}:=\alpha_{k, a}$. The intersection pairings $\left(\alpha_{a^{\prime}} \mid \alpha_{b^{\prime}}\right)$ are straightforward to compute using the formula from above. We get that $\left(\alpha_{a^{\prime}} \mid \alpha_{b^{\prime}}\right)$ coincides with the $\left(a^{\prime}, b^{\prime}\right)$-entry of the following matrix:

$$
\left(\begin{array}{cccccc}
2 & -1 & 0 & -1 & 0 & 0 \\
-1 & 2 & -1 & 1 & -1 & 0 \\
0 & -1 & 2 & 0 & 1 & -1 \\
-1 & 1 & 0 & 2 & -1 & 0 \\
0 & -1 & 1 & -1 & 2 & -1 \\
0 & 0 & -1 & 0 & -1 & 2
\end{array}\right)
$$

The above matrix has determinant 3. Therefore, the set $\left\{\alpha_{k, a} \mid 1 \leq a \leq 2,1 \leq k \leq 3\right\}$ is a set of linearly independent vanishing cycles. Since the set of all vanishing cycles is a root system of type $E_{6}$ and the determinant of the Cartan matrix of a root system of $E_{6}$ is also 3 , we get that the $\left\{\alpha_{k, a}\right\}$ is a set of simple roots. In particular, it is a $\mathbb{Z}$-basis of the Milnor lattice.

\section{$2.6 \quad E_{7}$-singularity}

Let us fix the following basis of $H_{f}$ :

$$
\phi_{i}= \begin{cases}x_{1}^{i-1} & \text { if } 1 \leqslant i \leqslant 3 \\ x_{2} x_{1}^{i-4} & \text { if } 4 \leqslant i \leqslant 6 \\ x_{2}^{2} & \text { if } i=7\end{cases}
$$

The residue pairing takes the form

$$
\left(\phi_{i}, \phi_{j}\right)= \begin{cases}\frac{1}{h} \delta_{i+j, 7}, & 1 \leqslant i, j \leqslant 6, \\ -\frac{1}{6}, & i=j=7, \\ 0, & \text { otherwise }\end{cases}
$$

where $h=18$ is the Coxeter number. The Riemann surface $M_{\mu}$ for $\mu \neq 0$ is a non-singular curve in $\mathbb{C}^{2}$ defined by the equation $x_{1}^{3}+x_{1} x_{2}^{3}=\mu$. The projection $\left(x_{1}, x_{2}\right) \mapsto x_{1}$ defines a degree 3 branched covering $M_{\mu} \rightarrow \mathbb{C}^{*}$, with branching points $x_{1, k}=\mu^{\frac{1}{3}} \eta_{3}^{k}(0 \leqslant k \leqslant 2)$, where $\eta_{3}=e^{\frac{2}{3} \pi \mathbf{i}}$.

The method of constructing loops in $M_{\mu}$ is similar to that of $D_{N}$-singularity. Let $A_{k}^{\prime}$ be a simple loop in $\mathbb{C}^{*}$ around the line segment $L_{k}:=\left[0, x_{1, k}\right]$, that is, $A_{k}^{\prime}$ is a loop starting at a point $\tilde{\epsilon} \eta_{3}^{k}(0<\tilde{\epsilon} \ll 1)$ on the line segment $L_{k}$ sufficiently close to 0 , it goes along the line segment $L_{k}$, just before hitting the branch point $x_{1, k}$ it makes a small loop $C_{k}$ counterclockwise around it, it returns back to the starting point along the line segment $L_{k}$, and finally it makes a small loop $C_{0}$ counterclockwise around 0. Clearly, the loop $A_{k}^{\prime}=C_{0} \circ L_{k}^{-1} \circ C_{k} \circ L_{k}$ lifts to a loop $A_{k, a}, a=0,1,2$ in $M_{\mu}$, where $a$ indicates the lift of the base point, that is, the base point

is lifted to $\left(x_{1, k, a}=\widetilde{\epsilon} \eta_{3}^{k}, x_{2, k, a}=\left(\frac{\mu-\widetilde{\epsilon}^{3}}{\widetilde{\epsilon}}\right)^{\frac{1}{3}} \eta_{3}^{a-\frac{k}{3}}\right)$. We will compute the period integrals along $A_{k, a}$ for $0 \leq k, a \leq 2$. As a biproduct of our computation we will get that the following set of 7 loops $\left\{A_{0,1}, A_{1,1}, A_{2,1}, A_{0,2}, A_{1,2}, A_{2,2}, A_{0,0}\right\}$ represents a basis of $H_{1}\left(M_{\mu} ; \mathbb{Z}\right)$.

Let us compute the periods of the holomorphic forms

$$
\phi_{i}\left(x_{1}, x_{2}\right) \frac{d x_{1} d x_{2}}{d g}= \begin{cases}-\frac{x_{1}^{i-2} d x_{1}}{3 x_{2}^{2}} & \text { if } 1 \leq i \leq 3, \\ -\frac{x_{1}^{i-5}}{3 x_{2}} d x_{1} & \text { if } 4 \leq i \leq 6, \\ -\frac{d x_{1}}{3 x_{1}} & \text { if } i=7,\end{cases}
$$


along the cycle $A_{k, a}$. Let us parametrize $A_{k}^{\prime}$ as follows:

$$
\begin{aligned}
& C_{0}: x_{1}=\tilde{\epsilon} e^{\mathrm{i} \theta}, \quad \frac{2 k}{3} \pi \leq \theta \leq \frac{2 k+6}{3} \pi, \\
& L_{k}: x_{1}=\eta_{3}^{k} \mu^{\frac{1}{3}} t^{\frac{1}{3}}, \quad \frac{\epsilon^{3}}{\mu} \leqslant t \leqslant\left(1-\frac{\epsilon}{\mu^{\frac{1}{3}}}\right)^{3}, \\
& C_{k}: x_{1}=\eta_{3}^{k} \mu^{\frac{1}{3}}+\epsilon e^{\mathrm{i} \theta}, \quad \frac{2 k-3}{3} \pi \leqslant \theta \leqslant \frac{2 k+3}{3} \pi .
\end{aligned}
$$

The integrals along the lifts of $C_{0}$ and $C_{k}$ contribute to the period integral terms of orders respectively

$$
\begin{cases}O\left(\tilde{\epsilon}^{i-1+\frac{2}{3}}\right) \text { and } O\left(\epsilon^{1-\frac{2}{3}}\right) & \text { if } 1 \leq i \leq 3, \\ O\left(\tilde{\epsilon}^{i-4+\frac{1}{3}}\right) \text { and } O\left(\epsilon^{1-\frac{1}{3}}\right) & \text { if } 4 \leq i \leq 6, \\ O\left(\tilde{\epsilon}^{0}\right) \text { and } O\left(\epsilon^{1}\right) & \text { if } i=7 .\end{cases}
$$

In the limit $\tilde{\epsilon}, \epsilon \rightarrow 0$ all integrals along the loops $C_{0}$ and $C_{k}$ vanish except for the integral along $C_{0}$ when $i=7$. The latter however is straightforward to compute. Therefore, after passing to the limit $\epsilon, \tilde{\epsilon} \rightarrow 0$, we get

$$
\int_{A_{k, a}} \phi_{i}\left(x_{1}, x_{2}\right) \frac{d x_{1} d x_{2}}{d g}= \begin{cases}\left(1-\eta_{3}^{-2}\right) \int_{L_{k, a}} \frac{-\phi_{i} d x_{1}}{3 x_{1} x_{2}^{2}} & \text { if } 1 \leq i \leq 3, \\ \left(1-\eta_{3}^{-1}\right) \int_{L_{k, a}}^{\frac{-\phi_{i} d x_{1}}{3 x_{1} x_{2}^{2}}} & \text { if } 4 \leq i \leq 6, \\ -\frac{2 \pi \mathbf{i}}{3} & \text { if } i=7,\end{cases}
$$

where $\eta_{3}:=e^{\frac{2 \pi \mathbf{i}}{3}}$ and the integral

$$
\int_{L_{k, a}} \frac{-\phi_{i} d x_{1}}{3 x_{1} x_{2}^{2}}=\left\{\begin{array}{cl}
\int_{0}^{1} \frac{-\eta_{3}^{k\left(i-\frac{1}{3}\right)-2 a} \mu^{\frac{1}{3}\left(i-\frac{7}{3}\right)} t^{\frac{1}{3}\left(i-\frac{10}{3}\right)} d t}{9(1-t)^{\frac{2}{3}}} & \\
=-\frac{\eta_{3}^{k\left(i-\frac{1}{3}\right)-2 a} \mu^{\frac{1}{3}\left(i-\frac{7}{3}\right)}}{9} B\left(\frac{i}{3}-\frac{1}{9}, \frac{1}{3}\right) & \text { if } 1 \leqslant i \leqslant 3, \\
\int_{0}^{1} \frac{-\eta_{3}^{k\left(i-4+\frac{1}{3}\right)-a} \mu^{\frac{1}{3}\left(i-4-\frac{2}{3}\right)} t^{\frac{1}{3}\left(i-4-\frac{8}{3}\right)} d t}{9(1-t)^{\frac{1}{3}}} & \\
=-\frac{\eta_{3}^{k\left(i-4+\frac{1}{3}\right)-a} \mu^{\frac{1}{3}\left(i-4-\frac{2}{3}\right)}}{9} B\left(\frac{i-4}{3}+\frac{1}{9}, \frac{2}{3}\right) & \text { if } 4 \leqslant i \leqslant 6 .
\end{array}\right.
$$

Let $\alpha_{k, a}=\Sigma A_{k, a}$ be the suspension. Recalling formula (2.1) and using (2.4)

$$
\begin{aligned}
& \left(I_{\alpha_{k, a}}^{(-1)}(\lambda), \phi_{i}\right):=\frac{1}{2 \pi} \int_{\alpha_{k, a}} \phi_{i} \frac{\omega}{d f}=\frac{1}{\pi} \partial_{\lambda} \int_{0}^{\lambda}(\lambda-\mu)^{\frac{1}{2}} \int_{A_{k, a}} \phi_{i}\left(x_{1}, x_{2}\right) \frac{d x_{1} d x_{2}}{d g} d \mu
\end{aligned}
$$

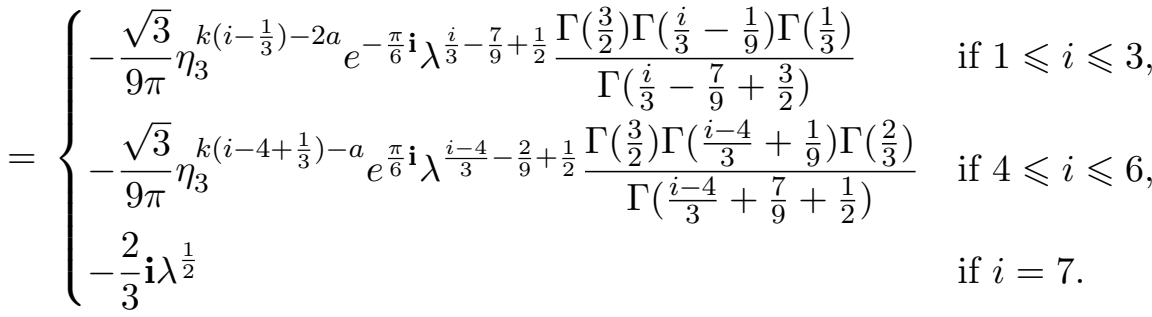


Recalling the formulas for the residue pairing in the basis $\left\{\phi_{i}\right\}$ we get

$$
I_{\alpha_{k, a}}^{(-1)}(\lambda)=h \sum_{i=1}^{6}\left(I_{\alpha_{k, a}}^{(-1)}(\lambda), \phi_{7-i}\right) \phi_{i}+4 \mathbf{i} \lambda^{\frac{1}{2}} \phi_{7} .
$$

Recalling formula (1.1) and using that

$$
\theta\left(\phi_{i}\right)= \begin{cases}\left(\frac{4}{9}-\frac{i-1}{3}\right) \phi_{i} & \text { if } 1 \leqslant i \leqslant 3 \\ \left(\frac{2}{9}-\frac{i-4}{3}\right) \phi_{i} & \text { if } 4 \leqslant i \leqslant 6 \\ 0 & \text { if } i=7,\end{cases}
$$

we get

$$
\begin{aligned}
\Psi\left(\alpha_{k, a}\right)= & -\sqrt{\frac{3}{\pi}} \sum_{i=1}^{3} e^{\frac{\pi}{6} \mathbf{i}} \eta_{3}^{k\left(\frac{1}{3}-i\right)-a} \Gamma\left(\frac{3-i}{3}+\frac{1}{9}\right) \Gamma\left(\frac{2}{3}\right) \phi_{i} \\
& -\sqrt{\frac{3}{\pi}} \sum_{i=4}^{6} e^{-\frac{\pi}{6} \mathbf{i}} \eta_{3}^{k\left(4-i-\frac{1}{3}\right)-2 a} \Gamma\left(\frac{7-i}{3}-\frac{1}{9}\right) \Gamma\left(\frac{1}{3}\right) \phi_{i}+2 \sqrt{-\pi} \phi_{7} .
\end{aligned}
$$

Recalling formula (2.2), we get the following formulas for the classical monodromy operator:

$$
\sigma\left(\alpha_{k, a}\right)=-\alpha_{k+1, a+1}, \quad k, a \in \mathbb{Z}_{3} .
$$

Using formula (2.3), we get that the intersection pairing

$$
\begin{aligned}
\left(\alpha_{k, a} \mid \alpha_{l, b}\right) & =\frac{1}{\pi}\left(\Psi\left(\alpha_{k, a}\right), \cos (\pi \theta) \Psi\left(\alpha_{l, b}\right)\right) \\
& =\frac{2}{3}+\frac{1}{6} \sum_{i=1}^{3}\left(\eta_{3}^{b-a+(l-k)\left(i-\frac{1}{3}\right)}+\eta_{3}^{a-b+(k-l)\left(i-\frac{1}{3}\right)}\right) \frac{\cos \left(\left(\frac{i}{3}-\frac{7}{9}\right) \pi\right)}{\sin \left(\left(\frac{i}{3}-\frac{1}{9}\right) \pi\right) \sin \left(\frac{\pi}{3}\right)} \\
& =\frac{2}{3}+\frac{1}{3} \sum_{i=1}^{3} \cos \left(\frac{2 \pi}{3}\left(a-b+(k-l)\left(i-\frac{1}{3}\right)\right)\right) \frac{\cos \left(\left(\frac{i}{3}-\frac{7}{9}\right) \pi\right)}{\sin \left(\left(\frac{i}{3}-\frac{1}{9}\right) \pi\right) \sin \left(\frac{\pi}{3}\right)} .
\end{aligned}
$$

Let us identify $\mathbb{Z}_{3}=\{0,1,2\}$. Every $1 \leq a^{\prime} \leq 7$ can be written uniquely in the form $a^{\prime}=$ $3(a-1)+k+1,1 \leq a \leq 3,0 \leq k \leq 2$. Put $\alpha_{a^{\prime}}:=\alpha_{k, \bar{a}}$, where $0 \leq \bar{a} \leq 2$ is the remainder of $a$ modulo 3 . Using the above formula, we get that the intersection pairing in the basis $\left\{\alpha_{a^{\prime}}\right\}_{1 \leq a^{\prime} \leq 7}$ takes the form

$$
\left(\begin{array}{lllllll}
2 & 1 & 1 & 0 & 1 & 1 & 0 \\
1 & 2 & 1 & 0 & 0 & 1 & 1 \\
1 & 1 & 2 & 0 & 0 & 0 & 1 \\
0 & 0 & 0 & 2 & 1 & 1 & 0 \\
1 & 0 & 0 & 1 & 2 & 1 & 0 \\
1 & 1 & 0 & 1 & 1 & 2 & 0 \\
0 & 1 & 1 & 0 & 0 & 0 & 2
\end{array}\right) .
$$

The above matrix has determinant 2. Since the determinant of the Cartan matrix of the root system of type $E_{7}$ is also 2, the conclusion is the same as in the case of $E_{6}$-singularity, that is, the cycles $\left(\alpha_{1}, \ldots, \alpha_{7}\right)=\left(\alpha_{0,1}, \alpha_{1,1}, \alpha_{2,1}, \alpha_{0,2}, \alpha_{1,2}, \alpha_{2,2}, \alpha_{0,0}\right)$ form a $\mathbb{Z}$-basis of the Milnor lattice and hence their images under $\Psi$, computed by formula (2.8), give a $\mathbb{Z}$-basis for the image of the Milnor lattice in $H_{f}$. 


\section{$2.7 \quad E_{8}$-singularity}

Let us fix the following basis of $H_{f}$ :

$$
\phi_{i}= \begin{cases}x_{2}^{i-1} & \text { if } 1 \leqslant i \leqslant 4, \\ x_{1} x_{2}^{i-5} & \text { if } 5 \leqslant i \leqslant 8 .\end{cases}
$$

The residue pairing takes the form

$$
\left(\phi_{i}, \phi_{j}\right)=\frac{1}{h} \delta_{i+j, 9}, \quad 1 \leqslant i, j \leqslant 8,
$$

where $h=30$ is the Coxeter number. The Riemann surface $M_{\mu}$ for $\mu \neq 0$ is a non-singular curve in $\mathbb{C}^{2}$ defined by equation $x_{1}^{3}+x_{2}^{5}=\mu$. The projection $\left(x_{1}, x_{2}\right) \mapsto x_{2}$ defines a degree 3 branched covering $M_{\mu} \rightarrow \mathbb{C}$, with branching points $x_{2, k}=\mu^{\frac{1}{5}} \eta_{5}^{k}, k \in \mathbb{Z}_{5}$, where $\eta_{5}=e^{\frac{2}{5} \pi \mathbf{i}}$.

The method for constructing loops in $M_{\mu}$ is almost the same as that for $E_{6}$-singularity. Let us omit the similar narration, i.e., we define the loops $A_{k, a}$ in the same way, except that now $a \in \mathbb{Z}_{3} \backslash\{0\}$ and $k \in \mathbb{Z}_{5} \backslash\{0\}$. We will see that the homology classes of these 8 loops form a $\mathbb{Z}$-basis of $H_{1}\left(M_{\mu} ; \mathbb{Z}\right)$. Let us compute the periods of the holomorphic forms

$$
\phi_{i}\left(x_{1}, x_{2}\right) \frac{d x_{1} d x_{2}}{d g}= \begin{cases}\frac{x_{2}^{i-1} d x_{2}}{3 x_{1}^{2}} & \text { if } 1 \leqslant i \leqslant 4, \\ \frac{x_{2}^{i-5} d x_{2}}{3 x_{1}} & \text { if } 5 \leqslant i \leqslant 8\end{cases}
$$

along the cycle $A_{k, a}$. Let us parametrize $A_{k}^{\prime}$ as follows:

$$
\begin{aligned}
& L_{k}: x_{2}=\eta_{5}^{k} \mu^{\frac{1}{5}} t^{\frac{1}{5}}, \quad 0 \leqslant t \leqslant\left(1-\frac{\epsilon}{\mu^{\frac{1}{5}}}\right)^{5}, \\
& C_{k}: x_{2}=\eta_{5}^{k} \mu^{\frac{1}{5}}+\epsilon e^{\mathrm{i} \theta}, \quad \frac{2 k-5}{5} \pi \leqslant \theta \leqslant \frac{2 k+5}{5} \pi .
\end{aligned}
$$

The integrals along the lifts of $C_{k}$ contribute to the period integral terms of orders $O\left(\epsilon^{\frac{1}{3}}\right)$ if $1 \leqslant i \leqslant 3$ and $O\left(\epsilon^{\frac{2}{3}}\right)$ if $4 \leqslant i \leqslant 6$. These terms vanish in the limit $\epsilon \rightarrow 0$. Therefore, under this limit, the periods of the holomorphic forms

$$
\int_{A_{k, a}} \phi_{i}\left(x_{1}, x_{2}\right) \frac{d x_{1} d x_{2}}{d g}=\left\{\begin{array}{l}
\left(1-\eta_{3}^{-2}\right)\left(\int_{L_{k, a}}-\int_{L_{k+1, a}}\right) \frac{\phi_{i} d x_{2}}{3 x_{1}^{2}} \quad \text { if } 1 \leqslant i \leqslant 4 \\
\left(1-\eta_{3}^{-1}\right)\left(\int_{L_{k, a}}-\int_{L_{k+1, a}}\right) \frac{\phi_{i} d x_{2}}{3 x_{1}^{2}} \quad \text { if } 5 \leqslant i \leqslant 8
\end{array}\right.
$$

where $\eta_{3}:=e^{\frac{2 \pi \mathbf{i}}{3}}$ and the integral

$$
\int_{L_{k, a}} \frac{\phi_{i} d x_{2}}{3 x_{1}^{2}}= \begin{cases}\int_{0}^{1} \frac{\eta_{5}^{k i} \mu^{\frac{i}{5}} t^{\frac{i}{5}-1} d t}{15 \mu^{\frac{2}{3}}(1-t)^{\frac{2}{3}} \eta_{3}^{2 a}}=\frac{\eta_{5}^{k i}}{15} \eta_{3}^{a} \mu^{\frac{i}{5}-\frac{2}{3}} B\left(\frac{i}{5}, \frac{1}{3}\right) & \text { if } 1 \leqslant i \leqslant 4 \\ \int_{0}^{1} \frac{\eta_{5}^{k(i-4)} \mu^{\frac{i-4}{5}} t^{\frac{i-4}{5}}-1}{15 \mu^{\frac{1}{3}}(1-t)^{\frac{1}{3}} \eta_{3}^{a}}=\frac{\eta_{5}^{k(i-4)}}{15} \eta_{3}^{2 a} \mu^{\frac{i-4}{5}-\frac{1}{3}} B\left(\frac{i-4}{5}, \frac{2}{3}\right) & \text { if } 5 \leqslant i \leqslant 8\end{cases}
$$

Then,

$$
\int_{A_{k, a}} \phi_{i}\left(x_{1}, x_{2}\right) \frac{d x_{1} d x_{2}}{d g}
$$




$$
= \begin{cases}\left(1-\eta_{3}^{-2}\right)\left(1-\eta_{5}^{i}\right) \frac{\eta_{5}^{k i}}{15} \eta_{3}^{a} \mu^{\frac{i}{5}-\frac{2}{3}} B\left(\frac{i}{5}, \frac{1}{3}\right) & \text { if } 1 \leqslant i \leqslant 4, \\ \left(1-\eta_{3}^{-1}\right)\left(1-\eta_{5}^{i-4}\right) \frac{\eta_{5}^{k(i-4)}}{15} \eta_{3}^{2 a} \mu^{\frac{i-4}{5}-\frac{1}{3}} B\left(\frac{i-4}{5}, \frac{2}{3}\right) & \text { if } 5 \leqslant i \leqslant 8 .\end{cases}
$$

Let $\alpha_{k, a}=\Sigma A_{k, a}$ be the suspension. Recalling formula (2.1) and using (2.4)

$$
\begin{aligned}
& \left(I_{\alpha_{k, a}}^{(-1)}(\lambda), \phi_{i}\right):=\frac{1}{2 \pi} \int_{\alpha_{k, a}} \phi_{i} \frac{\omega}{d f}=\frac{1}{\pi} \partial_{\lambda} \int_{0}^{\lambda}(\lambda-\mu)^{\frac{1}{2}} \int_{A_{k, a}} \phi_{i}\left(x_{1}, x_{2}\right) \frac{d x_{1} d x_{2}}{d g} d \mu \\
& = \begin{cases}\frac{\sqrt{3}}{15 \pi} \eta_{3}^{a} e^{-\frac{\pi}{6} \mathbf{i} \lambda^{\frac{i}{5}}-\frac{1}{6} \frac{\Gamma\left(\frac{3}{2}\right) \Gamma\left(\frac{i}{5}\right) \Gamma\left(\frac{1}{3}\right)}{\Gamma\left(\frac{i}{5}+\frac{5}{6}\right)} \eta_{5}^{k i}\left(1-\eta_{5}^{i}\right)} & \text { if } 1 \leqslant i \leqslant 4, \\
\frac{\sqrt{3}}{15 \pi} \eta_{3}^{2 a} e^{\frac{\pi}{6} \mathbf{i}} \lambda^{\frac{i-4}{5}+\frac{1}{6}} \frac{\Gamma\left(\frac{3}{2}\right) \Gamma\left(\frac{i-4}{5}\right) \Gamma\left(\frac{2}{3}\right)}{\Gamma\left(\frac{i-4}{5}+\frac{7}{6}\right)} \eta_{5}^{k(i-4)}\left(1-\eta_{5}^{i-4}\right) & \text { if } 5 \leqslant i \leqslant 8 .\end{cases}
\end{aligned}
$$

Recalling the formulas for residue pairing, we get

$$
I_{\alpha_{k, a}}^{(-1)}(\lambda)=h \sum_{i=1}^{8}\left(I_{\alpha_{k, a}}^{(-1)}(\lambda), \phi_{9-i}\right) \phi_{i} .
$$

By definition,

$$
\theta\left(\phi_{i}\right)= \begin{cases}\left(\frac{2}{3}-\frac{i}{5}\right) \phi_{i}, & \text { if } 1 \leqslant i \leqslant 4 \\ \left(\frac{1}{3}-\frac{i-4}{5}\right) \phi_{i}, & \text { if } 5 \leqslant i \leqslant 8\end{cases}
$$

Therefore, recalling formula (1.1), we get

$$
\begin{aligned}
\Psi\left(\alpha_{k, a}\right)= & \sqrt{\frac{3}{\pi}} \sum_{i=1}^{4} e^{\frac{\pi}{6} \mathbf{i}} \eta_{3}^{2 a} \Gamma\left(1-\frac{i}{5}\right) \Gamma\left(\frac{2}{3}\right) \eta_{5}^{-k i}\left(1-\eta_{5}^{-i}\right) \phi_{i} \\
& +\sqrt{\frac{3}{\pi}} \sum_{i=5}^{8} e^{-\frac{\pi}{6} \mathbf{i}} \eta_{3}^{a} \Gamma\left(1-\frac{i-4}{5}\right) \Gamma\left(\frac{1}{3}\right) \eta_{5}^{k(4-i)}\left(1-\eta_{5}^{4-i}\right) \phi_{i}
\end{aligned}
$$

Recalling formula (2.2), we get that the following formulas for the classical monodromy operator:

$$
\sigma\left(\alpha_{k, a}\right)=-\alpha_{k+1, a+1}, \quad k \in \mathbb{Z}_{5}, \quad a \in \mathbb{Z}_{3} .
$$

Recalling formula (2.3), the intersection pairing

$$
\begin{aligned}
\left(\alpha_{k, a} \mid \alpha_{l, b}\right) & =\frac{1}{\pi}\left(\Psi\left(\alpha_{k, a}\right), \cos (\pi \theta) \Psi\left(\alpha_{l, b}\right)\right) \\
& =\frac{1}{10} \sum_{i=1}^{4}\left(\eta_{3}^{b-a} \eta_{5}^{(l-k) i}+\eta_{3}^{a-b} \eta_{5}^{(k-l) i}\right) \frac{\cos \left(\left(\frac{i}{5}-\frac{2}{3}\right) \pi\right)}{\sin \left(\frac{i}{5} \pi\right) \sin \left(\frac{\pi}{3}\right)}\left(2-\eta_{5}^{-i}-\eta_{5}^{i}\right) \\
& =\frac{4}{5} \sum_{i=1}^{4} \cos \left(\frac{2}{3}(b-a) \pi+\frac{2 i}{5}(l-k) \pi\right) \frac{\cos \left(\left(\frac{i}{5}-\frac{2}{3}\right) \pi\right)}{\sin \left(\frac{\pi}{3}\right)} \sin \left(\frac{i}{5} \pi\right) .
\end{aligned}
$$

Let us identify $\mathbb{Z}_{3} \backslash\{0\}=\{1,2\}$ and $\mathbb{Z}_{5} \backslash\{0\}=\{1,2,3,4\}$. Every $1 \leq a^{\prime} \leq 8$ can be written uniquely in the form $a^{\prime}=4(a-1)+k$, where $1 \leq a \leq 2$ and $1 \leq k \leq 4$. Put $\alpha_{a^{\prime}}:=\alpha_{k, a}$. Then 
the intersection matrix $\left(\alpha_{a^{\prime}} \mid \alpha_{b^{\prime}}\right)$ takes the following form:

$$
\left(\begin{array}{cccccccc}
2 & -1 & 0 & 0 & -1 & 0 & 0 & 0 \\
-1 & 2 & -1 & 0 & 1 & -1 & 0 & 0 \\
0 & -1 & 2 & -1 & 0 & 1 & -1 & 0 \\
0 & 0 & -1 & 2 & 0 & 0 & 1 & -1 \\
-1 & 1 & 0 & 0 & 2 & -1 & 0 & 0 \\
0 & -1 & 1 & 0 & -1 & 2 & -1 & 0 \\
0 & 0 & -1 & 1 & 0 & -1 & 2 & -1 \\
0 & 0 & 0 & -1 & 0 & 0 & -1 & 2
\end{array}\right) .
$$

The above matrix has determinant 1. Since the determinant of the Cartan matrix of the root system of type $E_{8}$ is also 1, the conclusion is the same as in the previous cases.

\section{K-theoretic interpretation}

The goal of this section is to prove Theorem 1.3.

\subsection{Fermat cases}

Let us compute explicitly the map $\operatorname{ch}_{\Gamma}$ for $f(x)=f^{T}(x)=x_{1}^{a_{1}}+x_{2}^{a_{2}}+x_{3}^{a_{3}}$ with $a_{3}=2$. In fact, our computation works for arbitrary $a_{3}$ as well, except for one small technical detail, that is, we will prove that the group $K_{G^{T}}^{-1}\left(V_{1}^{T}\right)$ is torsion free. This fact should be true for any positive integer $a_{3}$, but the argument that we give works only if $a_{3}=2$. The group

$$
G^{T}=\left\{g=\left(g_{1}, g_{2}, g_{3}\right) \in\left(\mathbb{C}^{*}\right) \mid g_{1}^{a_{1}}=g_{2}^{a_{2}}=g_{3}^{a_{3}}=1\right\} .
$$

If $g \in G^{T}$ is such that $I=\left\{i \mid g_{i}=1\right\}$ is a non-empty set, then it is easy to see that the map $x=\left(x_{1}, x_{2}, x_{3}\right) \mapsto\left(x_{i}^{a_{i}}\right)_{i \in I}$ induces isomorphisms $\operatorname{Fix}_{g}\left(\mathbb{C}^{3}\right) / G^{T} \cong \mathbb{C}^{I}$ and $\operatorname{Fix}_{g}\left(V_{1}^{T}\right) / G^{T} \cong H_{I}$, where $H_{I} \subset \mathbb{C}^{I}$ is the hyperplane $\sum_{i \in I} y_{i}=1$. Since the pair $\left(\mathbb{C}^{I}, H_{I}\right)$ is contractible the groups

$$
K^{0}\left(\operatorname{Fix}_{g}\left(\mathbb{C}^{3}\right) / G^{T}, \operatorname{Fix}_{g}\left(V_{1}^{T}\right) / G^{T}\right)=H^{*}\left(\operatorname{Fix}_{g}\left(\mathbb{C}^{3}\right) / G^{T}, \operatorname{Fix}_{g}\left(V_{1}^{T}\right) / G^{T}\right)=0 .
$$

If $g \in G^{T}$ is such that $g_{i} \neq 1$ for all $i$, then $\operatorname{Fix}_{g}\left(\mathbb{C}^{3}\right)=\{0\}$ and $\operatorname{Fix}_{g}\left(V_{1}^{T}\right)=\varnothing$. Note that the number of such $g$ is $N=\left(a_{1}-1\right)\left(a_{2}-1\right)\left(a_{3}-1\right)$, that is, the multiplicity of the singularity corresponding to the polynomial $f$.

Lemma 3.1. The group $K_{G^{T}}^{-1}\left(V_{1}^{T}\right)$ is torsion free.

Let us postpone the proof of this lemma until Section 3.4. Note that $K_{G^{T}}^{-1}\left(V_{1}^{T}\right) \otimes \mathbb{C}=0$. Therefore, according to the above Lemma 3.1, we have $K_{G^{T}}^{-1}\left(V_{1}^{T}\right)=0$. The long exact sequence of the pair $\left(\mathbb{C}^{3}, V_{1}^{T}\right)$ yields the following exact sequence

$$
0 \longrightarrow K_{G^{T}}^{0}\left(\mathbb{C}^{3}, V_{1}^{T}\right) \longrightarrow K_{G^{T}}^{0}\left(\mathbb{C}^{3}\right) \longrightarrow K_{G^{T}}^{0}\left(V_{1}^{T}\right)
$$

On the other hand, $K_{G^{T}}^{0}\left(\mathbb{C}^{3}\right)$ coincides with the representation ring of $G^{T}$, that is,

$$
K_{G^{T}}^{0}\left(\mathbb{C}^{3}\right)=\mathbb{Z}\left[L_{1}, L_{2}, L_{3}\right] /\left(L_{1}^{a_{1}}-1, L_{2}^{a_{2}}-1, L_{3}^{a_{3}}-1\right),
$$

where $L_{i}=\mathbb{C}^{3} \times \mathbb{C}$ is the trivial bundle with $G^{T}$-action $g \cdot(x, \lambda):=\left(g x, g_{i} \lambda\right)$. Note that $T \mathbb{C}^{3} \cong L_{1}+L_{2}+L_{3}$ in the category of $G^{T}$-equivariant bundles. We claim that

$$
K_{G^{T}}^{0}\left(\mathbb{C}^{3}, V_{1}^{T}\right)=\left(L_{1}-1\right)\left(L_{2}-1\right)\left(L_{3}-1\right) \mathbb{Z}\left[L_{1}, L_{2}, L_{3}\right] /\left(L_{1}^{a_{1}}-1, L_{2}^{a_{2}}-1, L_{3}^{a_{3}}-1\right) .
$$


Indeed, note that $s_{i}(x)=\left(x, f_{x_{i}}\right)$ is a $G^{T}$-equivariant section of $L_{i}^{-1}$. The Koszul complex corresponding to the sequence $\left(s_{1}, s_{2}, s_{3}\right)$ has the form

$$
L_{1} L_{2} L_{3} \longrightarrow \bigoplus_{1 \leq i<j \leq 3} L_{i} L_{j} \longrightarrow \bigoplus_{1 \leq i \leq 3} L_{i} \longrightarrow \underline{\mathbb{C}},
$$

where $\underline{\mathbb{C}}$ is the trivial bundle with trivial $G^{T}$-action. The sequence $\left(s_{1}, s_{2}, s_{3}\right)$ is regular, so the corresponding Koszul complex is a resolution of the structure sheaf of the zero locus $\left\{s_{1}=s_{2}=\right.$ $\left.s_{3}=0\right\}$. The zero locus is $\{0\}$ and since $0 \notin V_{1}^{T}$ the restriction of the Koszul complex to $V_{1}^{T}$ is exact, i.e., the Koszul complex represents an element of $K_{G^{T}}^{0}\left(\mathbb{C}^{3}, V_{1}^{T}\right)$. This proves that the RHS of (3.1) is a $\mathbb{Z}$-submodule of the LHS. Note that both the LHS and the RHS of (3.1) are free $\mathbb{Z}$-modules of rank $N$. Therefore, the quotient of LHS by RHS is a finite Abelian group. In order to prove that the quotient is 0 , it is sufficient to prove that if $g \in K_{G^{T}}^{0}\left(\mathbb{C}^{3}\right)$ and $m g$ belongs to the RHS of (3.1) for some integer $m$, then $g$ belongs to the RHS of (3.1) too. The proof is straightforward so we leave it as an exercise.

Let us fix the following basis of $K_{G^{T}}^{0}\left(\mathbb{C}^{3}, V_{1}^{T}\right)$ :

$$
A_{m_{1}, m_{2}, m_{3}}:=L_{1}^{m_{1}} L_{2}^{m_{2}} L_{3}^{m_{3}}\left(L_{1}-1\right)\left(L_{2}-1\right)\left(L_{3}-1\right), \quad 0 \leq m_{i} \leq a_{i}-2 .
$$

Let $e_{k_{1}, k_{2}, k_{3}}=1 \in H^{0}\left(\operatorname{Fix}_{g}\left(\mathbb{C}^{3}\right) / G, \operatorname{Fix}_{g}\left(V_{1}^{T}\right) / G\right)$, where $g=\left(e^{2 \pi \mathbf{i} k_{1} / a_{1}}, e^{2 \pi \mathbf{i} k_{2} / a_{2}}, e^{2 \pi \mathbf{i} k_{3} / a_{3}}\right)$. We get

$$
\operatorname{ch}_{\Gamma}\left(A_{m_{1}, m_{2}, m_{3}}\right)=\frac{1}{2 \pi} \sum_{k_{1}=1}^{a_{1}-1} \sum_{k_{2}=1}^{a_{2}-1} \sum_{k_{3}=1}^{a_{3}-1} \prod_{i=1}^{3}\left(\Gamma\left(1-\frac{k_{i}}{a_{i}}\right) e^{-2 \pi \mathrm{i} k_{i} m_{i} / a_{i}}\left(e^{-2 \pi \mathrm{i} k_{i} / a_{i}}-1\right)\right) e_{k_{1}, k_{2}, k_{3}},
$$

where the ingredients of the above formula are computed as follows. Since Fix $\left(\mathbb{C}^{3}\right)=\{0\}$ and the action of $g$ on $\left.L_{i}\right|_{\operatorname{Fix}_{g}\left(\mathbb{C}^{3}\right)}$ is given by multiplication by $e^{2 \pi \mathbf{i} k_{i} / a_{i}}$ we get

$$
\begin{aligned}
& \left.\widehat{\Gamma}\left(L_{i}\right)\right|_{\operatorname{Fix}_{g}\left(\mathbb{C}^{3}\right) / G^{T}}=\Gamma\left(1-\frac{k_{i}}{a_{i}}\right) \quad \text { and } \\
& \left.\iota^{*} \widetilde{\operatorname{ch}}\left(L_{i}\right)\right|_{\operatorname{Fix}_{g}\left(\mathbb{C}^{3}\right) / G^{T}}=\left.\widetilde{\operatorname{ch}}\left(L_{i}^{-1}\right)\right|_{\operatorname{Fix}_{g}\left(\mathbb{C}^{3}\right) / G^{T}}=e^{-2 \pi \mathbf{i} k_{i} / a_{i}},
\end{aligned}
$$

where we used that $\iota^{*}\left(L_{i}\right)=L_{i}^{-1}$. The orbifold tangent bundle $\left[T \mathbb{C}^{3} / G^{T}\right]=L_{1}+L_{2}+L_{3}$ so its $\Gamma$-class is $\prod_{i=1}^{3} \widehat{\Gamma}\left(L_{i}\right)$, while $\left.\iota^{*} \widetilde{c h}\right|_{\operatorname{Fix}_{g}\left(\mathbb{C}^{3}\right) / G^{T}}$ is a ring homomorphism, so the computation of its value on $A_{m_{1}, m_{2}, m_{3}}$ amounts to the substitution $L_{i} \mapsto e^{-2 \pi \mathbf{i} k_{i} / a_{i}}$. Let us specialize the above formula to the cases of $A_{N}, E_{6}$, and $E_{8}$ singularities. In the first case $a_{1}=N+1, a_{2}=a_{3}=2$. The above formula takes the form

$$
\operatorname{ch}_{\Gamma}\left(A_{m, 0,0}\right)=2 \sum_{k=1}^{N} \eta^{-k m}\left(\eta^{-k}-1\right) \Gamma\left(1-\frac{k}{N+1}\right) e_{k, 1,1}
$$

Comparing with (2.5), we get that if we define $\operatorname{mir}\left(\phi_{i}\right)=e_{i, 1,1}(1 \leq i \leq N)$, then the images of $\Psi$ and $\operatorname{ch}_{\Gamma}$ will coincide. The vanishing cycle $\alpha_{k, a}$ corresponds to $(-1)^{a} A_{k, 0,0}$.

For the case of $E_{6}$ we have $a_{1}=3, a_{2}=4, a_{3}=2$. The formula takes the form

$$
\begin{aligned}
\operatorname{ch}_{\Gamma}\left(A_{m_{1}, m_{2}, 0}\right)= & -\frac{1}{\sqrt{\pi}} \sum_{k_{1}=1}^{2} \sum_{k_{2}=1}^{3} \Gamma\left(1-\frac{k_{1}}{3}\right) \Gamma\left(1-\frac{k_{2}}{4}\right) \\
& \times \eta_{3}^{-k_{1} m_{1}} \eta_{4}^{-k_{2} m_{2}}\left(\eta_{3}^{-k_{1}}-1\right)\left(\eta_{4}^{-k_{2}}-1\right) e_{k_{1}, k_{2}, 1},
\end{aligned}
$$


where $\eta_{3}=e^{2 \pi \mathbf{i} / 3}$ and $\eta_{4}=e^{2 \pi \mathbf{i} / 4}=\mathbf{i}$. Note that $\eta_{3}^{-1}-1=-\sqrt{3} e^{\pi \mathbf{i} / 6}$ and $\eta_{3}^{-2}-1=-\sqrt{3} e^{-\pi \mathbf{i} / 6}$. Comparing with (2.7) we get that if we define

$$
\operatorname{mir}\left(\phi_{i}\right)= \begin{cases}e_{1, i, 1}, & \text { for } 1 \leq i \leq 3 \\ e_{2, i-3,1}, & \text { for } 4 \leq i \leq 6,\end{cases}
$$

then the images of $\Psi$ and $\operatorname{ch}_{\Gamma}$ will coincide. The vanishing cycle $\alpha_{k, a}$ corresponds to $-A_{a, k, 0}$.

Suppose now that the singularity is of type $E_{8}$, that is, $a_{1}=3, a_{2}=5$, and $a_{3}=2$. The formula takes the form

$$
\begin{aligned}
\operatorname{ch}_{\Gamma}\left(A_{m_{1}, m_{2}, 0}\right)= & -\frac{1}{\sqrt{\pi}} \sum_{k_{1}=1}^{2} \sum_{k_{2}=1}^{4} \Gamma\left(1-\frac{k_{1}}{3}\right) \Gamma\left(1-\frac{k_{2}}{5}\right) \\
& \times \eta_{3}^{-k_{1} m_{1}} \eta_{5}^{-k_{2} m_{2}}\left(\eta_{3}^{-k_{1}}-1\right)\left(\eta_{5}^{-k_{2}}-1\right) e_{k_{1}, k_{2}, 1},
\end{aligned}
$$

where $\eta_{3}=e^{2 \pi \mathbf{i} / 3}$ and $\eta_{5}=e^{2 \pi \mathbf{i} / 5}$. Comparing with formula (2.9) we get that if we define

$$
\operatorname{mir}\left(\phi_{i}\right)= \begin{cases}e_{1, i, 1}, & \text { for } 1 \leq i \leq 4, \\ e_{2, i-4,1}, & \text { for } 5 \leq i \leq 8,\end{cases}
$$

then the images of $\Psi$ and $\operatorname{ch}_{\Gamma}$ will coincide. The vanishing cycle $\alpha_{k, a}$ corresponds to $-A_{a, k, 0}$.

\subsection{Suspension isomorphism}

Suppose that $X$ is a finite CW-complex equipped with an action of a finite (or more generally compact Lie) group $G$. Let $\mu_{2}=\{ \pm 1\}$ and

$$
\Sigma X=X \times\{-1\} \backslash X \times[-1,1] / X \times\{1\}
$$

be the suspension of $X$, where the double quotient simply means that the quotient is taken in two steps: first by, say, $X \times\{-1\}$ and then by $X \times\{1\}$. Note that $G \times \mu_{2}$ acts naturally on $\Sigma X$ via $(g, \epsilon) \cdot(x, t)=(g x, \epsilon t)$ and that the 0 -dimensional sphere $\mathbb{S}^{0}:=\Sigma X-X \times(-1,1)$ is a $G \times \mu_{2}$-equivariant subcomplex of $\Sigma X$.

Let $L=[-1,1] \times \mathbb{C}$ be the trivial $\mu_{2}$-equivariant line bundle on the interval $I:=[-1,1]$, where the representation of $\mu_{2}$ on $\mathbb{C}$ is given by $\epsilon \cdot \lambda=\epsilon \lambda$. It is an easy and amusing exercise to check that $K_{\mu_{2}}^{0}(I, \partial I)=\mathbb{Z} \ell$, where $\ell$ is the relative $\mathrm{K}$-theoretic class of the complex $L \stackrel{t}{\longrightarrow} \underline{\mathbb{C}}$, where $\underline{\mathbb{C}}=I \times \mathbb{C}$ is the trivial $\mu_{2}$-equivariant line bundle corresponding to the trivial representation of $\mu_{2}$ on $\mathbb{C}$ and the map is induced by $(t, \lambda) \mapsto(t, t \lambda)$.

Lemma 3.2. The exterior tensor product by $\ell$ induces an isomorphism

$$
K_{G}^{i}(X) \cong K_{G \times \mu_{2}}^{i}\left(\Sigma X, \mathbb{S}^{0}\right) .
$$

Proof. By definition

$$
K_{G \times \mu_{2}}^{i}\left(\Sigma X, \mathbb{S}^{0}\right)=\widetilde{K}_{G \times \mu_{2}}^{i}\left(\Sigma X / \mathbb{S}^{0}\right)=K_{G \times \mu_{2}}^{i}(X \times I, X \times \partial I) \cong K_{G}^{i}(X) \otimes K_{\mu_{2}}^{0}(I, \partial I),
$$

where we used that $K_{\mu_{2}}^{i}(I, \partial I)$ is isomorphic to $\mathbb{Z}$ for $i$ even and 0 for $i$ odd, so the last isomorphism is given by the equivariant Künneth formula (see [15]).

Suppose now that $Y \subset X$ is a $G$-invariant $C W$-subcomplex of $X$. Using the long exact sequence of the triple $\mathbb{S}^{0} \subset \Sigma Y \subset \Sigma X$ and Lemma 3.2, it is straightforward to prove the following corollary.

Corollary 3.3. The exterior tensor product by $\ell$ induces an isomorphism

$$
K_{G}^{i}(X, Y) \cong K_{G \times \mu_{2}}^{i}(\Sigma X, \Sigma Y) .
$$




\subsection{The relative K-ring for $D_{N}$-singularity}

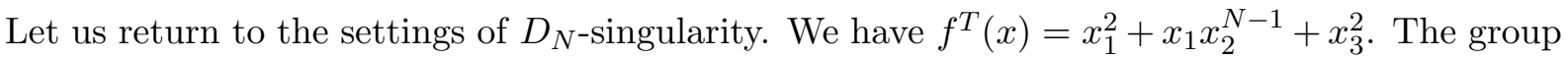
of diagonal symmetries of $f^{T}$ is

$$
G^{T}=\left\{t \in\left(\mathbb{C}^{*}\right)^{3} \mid t_{1}^{2}=t_{1} t_{2}^{N-1}=t_{3}^{2}=1\right\} .
$$

Let $L_{i}=\mathbb{C}^{3} \times \mathbb{C}$ be the $G^{T}$-equivariant line bundle for which the action of $G^{T}$ on $\mathbb{C}$ is given by the character $G^{T} \rightarrow \mathbb{C}^{*},\left(t_{1}, t_{2}, t_{3}\right) \mapsto t_{i}$. Let us introduce the following $N$ complexes of $G^{T}$-equivariant vector bundles on $\mathbb{C}^{3}$ :

$$
E_{i}^{\bullet}: \quad L_{1} L_{2}^{i-1} L_{3} \stackrel{d_{0}}{\longrightarrow} L_{1} L_{2}^{i-1} \oplus L_{2}^{i-1} L_{3} \stackrel{d_{1}}{\longrightarrow} L_{2}^{i-1}, \quad 1 \leq i \leq N-1,
$$

where the differentials are defined by $d_{0}(x, \lambda)=\left(x,-x_{3} \lambda, x_{1} \lambda\right)$ and $d_{1}\left(x, \lambda_{1}, \lambda_{3}\right)=\left(x, x_{1} \lambda_{1}+\right.$ $\left.x_{3} \lambda_{3}\right)$,

$$
E_{N}^{\bullet}: \quad L_{3} \stackrel{d_{0}}{\longrightarrow} \underline{\mathbb{C}} \oplus L_{3} \stackrel{d_{1}}{\longrightarrow} \underline{\mathbb{C}},
$$

where $d_{0}(x, \lambda)=\left(x,-x_{3} \lambda, x_{1}^{2} \lambda\right)$ and $d_{1}\left(x, \lambda_{1}, \lambda_{3}\right)=\left(x, x_{1}^{2} \lambda_{1}+x_{3} \lambda_{3}\right)$.

Proposition 3.4. The relative $K$-ring $K_{G^{T}}^{0}\left(\mathbb{C}^{3}, V^{T}\right) \cong \mathbb{Z}^{N}$ and the complexes $E_{i}^{\bullet}(1 \leq i \leq N)$ represent a $\mathbb{Z}$-basis.

Proof. Note that the complex $E_{i}^{\bullet}(1 \leq i \leq N-1)$ is a tensor product of $L_{2}^{i-1}, L_{1} \stackrel{x_{1}}{\longrightarrow} \underline{\mathbb{C}}$, and $L_{3} \stackrel{x_{3}}{\longrightarrow} \mathbb{C}$ and that the complex $E_{N}^{\bullet}$ is a tensor product of $\underline{\mathbb{C}} \stackrel{x_{1}^{2}}{\longrightarrow} \underline{\mathbb{C}}$ and $L_{3} \stackrel{x_{3}}{\longrightarrow} \underline{\mathbb{C}}$. On the other hand, we have $G^{T}=A \times \mu_{2}$, where $A=\left\{t \in\left(\mathbb{C}^{*}\right)^{2} \mid t_{1}^{2}=t_{1} t_{2}^{N-1}=1\right\}$. Recalling Corollary 3.3 we get $K_{G^{T}}^{0}\left(\mathbb{C}^{3}, V^{T}\right) \cong K_{A}^{0}\left(\mathbb{C}^{2}, M\right)$, where $M=\left\{x \in \mathbb{C}^{2} \mid x_{1}^{2}+x_{1} x_{2}^{N-1}=1\right\}$. Slightly abusing the notation we denote by $L_{1}$ and $L_{2}$ the restriction of the vector bundles $L_{1}$ and $L_{2}$ to $\mathbb{C}^{2}$. Note that the operation tensor product by the complex $L_{3} \stackrel{x_{3}}{\longrightarrow} \underline{\mathbb{C}}$ is precisely the exterior tensor product by the complex $\ell$ in the suspension isomorphism from Corollary 3.3. Therefore, it is sufficient to prove that the complexes $L_{1} L_{2}^{i-1} \stackrel{x_{1}}{\longrightarrow} L_{2}^{i-1}(1 \leq i \leq N-1)$ and $\underline{\mathbb{C}} \stackrel{x_{1}^{2}}{\longrightarrow} \mathbb{C}$ represent a $\mathbb{Z}$-basis of $K_{A}^{0}\left(\mathbb{C}^{2}, M\right)$.

The long exact sequence of the pair $\left(\mathbb{C}^{2}, M\right)$ yields the following exact sequence:

$$
0 \longrightarrow K_{A}^{-1}(M) \stackrel{\delta}{\longrightarrow} K_{A}^{0}\left(\mathbb{C}^{2}, M\right) \stackrel{\rho}{\longrightarrow} K_{A}^{0}\left(\mathbb{C}^{2}\right) \longrightarrow K_{A}^{0}(M),
$$

where we used that $K_{A}^{-1}\left(\mathbb{C}^{2}\right)=0$. We have

$$
K_{A}^{0}\left(\mathbb{C}^{2}\right)=\mathbb{Z}\left[L_{1}, L_{2}\right] /\left\langle L_{1}^{2}-1, L_{1} L_{2}^{N-1}-1\right\rangle,
$$

where the RHS is the representation ring of $A$. Just like in the Fermat cases it is easy to prove that the image of $\rho$ coincides with the ideal $\left(L_{1}-1\right) K_{A}^{0}\left(\mathbb{C}^{2}\right)$. Note that $\operatorname{Im}(\rho) \cong \mathbb{Z}^{N-1}$ and that $\rho\left(E_{i}^{\bullet}\right)=L_{2}^{i-1}\left(L_{1}-1\right)(1 \leq i \leq N-1)$ is a $\mathbb{Z}$-basis. It remains only to prove that $K_{A}^{-1}(M) \cong \mathbb{Z}$ and that $\operatorname{Im}(\delta)$ is generated as a $\mathbb{Z}$-module by the complex $E_{N}^{\bullet}$.

Let us first prove that $K_{A}^{-1}(M) \cong \mathbb{Z}$. Let $\pi: M \rightarrow \mathbb{C}^{*}$ be the $\operatorname{map}\left(x_{1}, x_{2}\right) \mapsto x_{1}^{2}$. The map $\pi$ is a branched covering with only one branch point, that is, $1 \in \mathbb{C}^{*}$. The corresponding ramification points are $R=\{(-1,0),(1,0)\}$. Note that $R$ is an $A$-invariant subset. The idea is to use the long exact sequence of the pair $(M, M \backslash R)$. The action of $A$ on $M \backslash R$ is free, so we have

$$
K_{A}^{i}(M \backslash R)=K^{i}((M \backslash R) / A)=K^{i}(\mathbb{C} \backslash\{0,1\}) .
$$


Therefore $K_{A}^{0}(M \backslash R) \cong \mathbb{Z}$ and $K_{A}^{-1}(M \backslash R) \cong \mathbb{Z}^{2}$. The groups $K^{i}(M, M \backslash R)$ are also easy to compute. Let $U \subset M$ be a small $A$-invariant open neighborhood of $R$. Then by excision $K_{A}^{i}(M, M \backslash R)=K_{A}^{i}(U, U \backslash R)$. Note that the open neighborhood $U$ can be identified with an open neighborhood of $R$ in the normal bundle $\nu_{R}$ to $R$ in $M$. Indeed, the normal bundle is trivial $\nu_{R}=R \times \mathbb{C}$ and a point in $\left(x_{1}, x_{2}\right) \in U$ satisfies $x_{1}=\frac{1}{2}\left(-x_{2}^{N-1} \pm \sqrt{x_{2}^{2 N-2}+4}\right)$, so the map $U \rightarrow \nu_{R},\left(x_{1}, x_{2}\right) \mapsto\left(( \pm 1,0), x_{2}\right)$ identifies $U$ with an open neighborhood of the zero section $R$ in $\nu_{R}$. Clearly, the pullback of $\nu_{R}$ to $U$ is $L_{2}$ and the Thom class of $\nu_{R}$ is represented as an element of $K_{A}^{0}\left(\nu_{R}\right)=K_{A}^{0}(U, U \backslash R)$ by the complex $\underline{\mathbb{C}} \stackrel{x_{2}}{\longrightarrow} L_{2}$. According to Thom isomorphism $K_{A}^{i}(U, U \backslash R) \cong K_{A}^{i}(R)$. Note that $R$ is an $A$-orbit, that is, $R=A / B$, where $B$ is the cyclic subgroup of $A$ generated by $\left(1, \eta^{2}\right)$. Therefore, $K_{A}^{-1}(R)=0$ and $K_{A}^{0}(R)$ coincides with the representation ring of $B$. Since the Thom isomorphism is given by tensor product with the Thom class, we get

$$
K_{A}^{0}(M, M \backslash R)=\bigoplus_{i=1}^{N-1} \mathbb{Z}\left[L_{2}^{i-1} \stackrel{x_{2}}{\longrightarrow} L_{2}^{i}\right] .
$$

The long exact sequence of the pair $(M, M \backslash R)$ takes the form

$$
0 \longrightarrow K_{A}^{-1}(M) \longrightarrow K_{A}^{-1}(M \backslash R) \stackrel{\delta}{\longrightarrow} K_{A}^{0}(M, M \backslash R) .
$$

We already proved that $K_{A}^{-1}(M \backslash R) \cong K^{-1}\left(\mathbb{C}^{2} \backslash\{0,1\}\right) \cong \mathbb{Z}^{2}$. We will make use of the following explicit interpretation of the $K$-group $K_{A}^{-1}$ ( ). By definition, for any finite CW-complex $X$, we have $K_{A}^{-1}(X)=\widetilde{K}_{A}^{0}(\Sigma(X \sqcup \mathrm{pt}))$. Since the complement of $X$ in $\Sigma(X \sqcup \mathrm{pt})$ is contractible, we can think of an element of $K_{A}^{-1}(X)$ as a representation of $A$ on some vector space $\mathbb{C}^{r}$ and an $A$ equivariant isomorphism $\phi: X \times \mathbb{C}^{r} \rightarrow X \times \mathbb{C}^{r}$, that is, an $A$-equivariant morphism $X \rightarrow \mathrm{GL}_{r}(\mathbb{C})$. In our case the elements of $K_{A}^{-1}(M \backslash R)$ are obtained by pullback from $K^{-1}(\mathbb{C} \backslash\{0,1\})$. The latter is generated by two elements that correspond, in the way described above, to the two maps $\mathbb{C} \backslash\{0,1\} \rightarrow \mathbb{C}^{*}, t \mapsto t$ and $t \mapsto 1-t$. Therefore, the group $K_{A}^{-1}(M \backslash R)$ is generated by the two elements that correspond to the two maps $M \backslash R \rightarrow \mathbb{C}^{*}$ defined by $\left(x_{1}, x_{2}\right) \mapsto x_{1}^{2}$ and $\left(x_{1}, x_{2}\right) \mapsto 1-x_{1}^{2}=x_{1} x_{2}^{N-1}$. The connecting morphism $\delta$ can be described as follows. Given an $A$-equivariant isomorphism $\phi:(M \backslash R) \times \mathbb{C}^{r} \rightarrow(M \backslash R) \times \mathbb{C}^{r}$, then let us pick an extension to a vector bundle morphism $\widetilde{\phi}: M \times \mathbb{C}^{r} \rightarrow M \times \mathbb{C}^{r}$. The resulting complex clearly represents an element of $K_{A}^{0}(M, M \backslash R)$ and that is what $\delta(\phi)$ is. The extensions in our case are straightforward to construct. We get that

$$
\operatorname{Im}(\delta)=\mathbb{Z}\left[\underline{\mathbb{C}} \stackrel{x_{1}^{2}}{\longrightarrow} \underline{\mathbb{C}}\right]+\mathbb{Z}\left[\underline{\mathbb{C}} \stackrel{x_{1} x_{2}^{N-1}}{\longrightarrow} \underline{\mathbb{C}}\right] .
$$

Note however, that $x_{1} \neq 0$ so $x_{1}^{2}$ defines an isomorphism, i.e., the first complex is 0 in $K_{A}^{0}(M, M \backslash R)$. In particular, the kernel of the connecting homomorphism $\delta$ is $\cong \mathbb{Z}$ and it is generated by the element in $K_{A}^{-1}(M \backslash R)$ corresponding to the map $M \backslash R \rightarrow \mathbb{C}^{*},\left(x_{1}, x_{2}\right) \rightarrow x_{1}^{2}$. This map extends to $M$, so we get that $K_{A}^{-1}(M) \cong \mathbb{Z}$ with generator corresponding to the map $M \rightarrow \mathbb{C}^{*},\left(x_{1}, x_{2}\right) \mapsto x_{1}^{2}$. Returning to the long exact sequence of the pair $\left(\mathbb{C}^{2}, M\right)$, we get that the connecting morphism $K_{A}^{-1}(M) \rightarrow K_{A}^{0}\left(\mathbb{C}^{2}, M\right)$ maps the generator of $K_{A}^{-1}(M)$ to the complex $\underline{\mathbb{C}} \stackrel{x_{1}^{2}}{\longrightarrow} \mathbb{C}$. This completes the proof of the proposition.

\subsection{Proof of Lemma 3.1}

We follow the same strategy as in the proof of Proposition 3.4. Let us denote by $M \subset \mathbb{C}^{2}$ the Riemann surface defined by $x_{1}^{a_{1}}+x_{2}^{a_{2}}=1$. Let $A=\left\{t \in\left(\mathbb{C}^{*}\right)^{2} \mid t_{1}^{a_{1}}=t_{2}^{a_{2}}=1\right\}$. Since 
$K_{G^{T}}^{-1}\left(V_{1}^{T}\right) \cong K_{A}^{-1}(M)$, we get that it is sufficient to prove that $K_{A}^{-1}(M)$ is torsion free. Let $\pi: M \rightarrow \mathbb{C}$ be the map $\left(x_{1}, x_{2}\right) \mapsto x_{1}^{a_{1}}$. The map $\pi$ is a branched covering with only one branching point, that is, $1 \in \mathbb{C}$. The corresponding ramification points are $R=\left\{(\xi, 0) \mid \xi^{a_{1}}=1\right\}$. The torsion freeness can be deduced easily from the long exact sequence of the pair $(M, M \backslash R)$. The action of $A$ on $M \backslash R$ is free, so we have

$$
K_{A}^{-1}(M \backslash R)=K^{-1}((M \backslash R) / A)=K^{-1}(\mathbb{C} \backslash\{1\}) \cong \mathbb{Z} .
$$

Using the Thom isomorphism for the normal bundle to $R$ in $M$, we get $K_{A}^{-1}(M, M \backslash R)=$ $K_{A}^{-1}(R)$. On the other hand, note that $R$ is the orbit of $A$ through the point $(1,0) \in M$, we get $R=A / B$, where $B \subset A$ is the cyclic subgroup generated by $\left(1, \eta_{a_{2}}\right), \eta_{a_{2}}=e^{2 \pi \mathbf{i} / a_{2}}$. Therefore, $K_{A}^{-1}(R)=K_{A}^{-1}(A / B)=K^{-1}(B)=0$. Recalling the long exact sequence of the pair $(M, M \backslash R)$, we get

$$
0 \longrightarrow K_{A}^{-1}(M) \longrightarrow K_{A}^{-1}(M \backslash R) \stackrel{\delta}{\longrightarrow} K_{A}^{0}(M, M \backslash R) .
$$

We get that $K_{A}^{-1}(M)$ can be embedded as a subgroup of $K_{A}^{-1}(M \backslash R) \cong \mathbb{Z}$. The latter is torsion free, so $K_{A}^{-1}(M)$ must be also torsion free.

Remark 3.5. The above argument can be continued to give a direct proof of the fact that $K_{A}^{-1}(M)=0$. Namely, using the Thom isomorphism, we can prove that the group $K_{A}^{0}(M, M \backslash R)$ is a free Abelian group of rank $a_{2}$ and that the complexes $\left[L_{2}^{i-1} \stackrel{x_{2}}{\longrightarrow} L_{2}^{i}\right] \quad\left(1 \leq i \leq a_{2}\right)$ represent a $\mathbb{Z}$-basis. Moreover, the image of the connecting morphism $\delta$ in (3.2) can be computed explicitly as well, that is, it coincides with the sum of the above complexes. In particular, we get that $\delta$ is an injective map, and hence $K_{A}^{-1}(M)=0$.

Remark 3.6. The long exact sequences of the pairs $(M, M \backslash R)$ and $\left(\mathbb{C}^{2}, M\right)$ can be computed explicitly, that is, both the groups and the differentials can be determined. This allows us to give an alternative proof of formula (3.1). We leave the details to the interested reader.

\subsection{The relative $\mathrm{K}$-ring for $\boldsymbol{E}_{7}$-singularity}

The argument from the previous section works also for $E_{7}$-singularity. Let us only state the result. The proof is completely analogous.

We have $f^{T}(x)=x_{1}^{3} x_{2}+x_{2}^{3}+x_{3}^{2}$. The group of diagonal symmetries of $f^{T}$ is

$$
G^{T}=\left\{t \in\left(\mathbb{C}^{*}\right)^{3} \mid t_{1}^{3} t_{2}=t_{2}^{3}=t_{3}^{2}=1\right\} .
$$

Let $L_{i}=\mathbb{C}^{3} \times \mathbb{C}$ be the $G^{T}$-equivariant line bundle for which the action of $G^{T}$ on $\mathbb{C}$ is given by the character $G^{T} \rightarrow \mathbb{C}^{*},\left(t_{1}, t_{2}, t_{3}\right) \mapsto t_{i}$. Let us introduce the following 7 complexes of $G^{T}$-equivariant vector bundles on $\mathbb{C}^{3}$ :

$$
E_{i}^{\bullet}: \quad L_{1}^{i-1} L_{3}^{-1} \stackrel{d_{0}}{\longrightarrow} L_{1}^{i-1} \oplus L_{1}^{i-1} L_{2} L_{3}^{-1} \stackrel{d_{1}}{\longrightarrow} L_{1}^{i-1} L_{2}, \quad 1 \leq i \leq 6,
$$

where the differentials are defined by $d_{0}(x, \lambda)=\left(x,-x_{3} \lambda, x_{2} \lambda\right)$ and $d_{1}\left(x, \lambda_{2}, \lambda_{3}\right)=\left(x, x_{2} \lambda_{2}+\right.$ $\left.x_{3} \lambda_{3}\right)$,

$$
E_{7}^{\bullet}: \quad L_{3} \stackrel{d_{0}}{\longrightarrow} \mathbb{C} \oplus L_{3} \stackrel{d_{1}}{\longrightarrow} \underline{\mathbb{C}},
$$

where $d_{0}(x, \lambda)=\left(x,-x_{3} \lambda, x_{2}^{3} \lambda\right)$ and $d_{1}\left(x, \lambda_{2}, \lambda_{3}\right)=\left(x, x_{2}^{3} \lambda_{2}+x_{3} \lambda_{3}\right)$.

Proposition 3.7. Let $V^{T}=\left\{x \in \mathbb{C}^{3} \mid f^{T}(x)=1\right\}$. The relative $K$-ring $K_{G^{T}}^{0}\left(\mathbb{C}^{3}, V^{T}\right) \cong \mathbb{Z}^{7}$ and the complexes $E_{i}^{\bullet}(1 \leq i \leq 7)$ represent a $\mathbb{Z}$-basis. 


\section{6 $\quad \Gamma$-integral structure for $D_{N}$-singularity}

Let us compute $\operatorname{ch}_{\Gamma}\left(E_{i}^{\bullet}\right)$ for $1 \leq i \leq N$. After a straightforward computation we get that the relative cohomology group $H\left(\operatorname{Fix}_{g}\left(\mathbb{C}^{3}\right), \operatorname{Fix}_{g}\left(V^{T}\right)\right)^{G^{T}}$ is not zero only in the following two cases: 1) $g=\left(g_{1}, g_{2}, g_{3}\right)$ with $g_{i} \neq 1$ for all $i$ and 2) $g=(1,1,-1)$. For the first case, there are $N-1$ elements, that is, $g=\left(-1, \eta^{2 i-1},-1\right)(1 \leq i \leq N-1)$ and the fixed point subsets are $\operatorname{Fix}_{g}\left(\mathbb{C}^{3}\right)=\{0\}$ and $\operatorname{Fix}_{g}\left(V^{T}\right)=\varnothing$. Therefore, $H\left(\operatorname{Fix}_{g}\left(\mathbb{C}^{3}\right), \operatorname{Fix}_{g}\left(V^{T}\right) ; \mathbb{C}\right)^{G^{T}} \cong \mathbb{C}$ is non-trivial only in degree 0 and we denote by $e_{i}:=1$ the unit of the cohomology group. For the second case, $\operatorname{Fix}_{g}\left(\mathbb{C}^{3}\right)=\mathbb{C}^{2}$ and $\operatorname{Fix}_{g}\left(V^{T}\right)=M=\left\{x_{1}^{2}+x_{1} x_{2}^{N-1}=1\right\}$. The relative cohomology group $H^{i}\left(\mathbb{C}^{2}, M ; \mathbb{C}\right)^{G^{T}} \cong H^{i-1}\left(M / G^{T} ; \mathbb{C}\right)$ for $i>0$ and $=0$ for $i=0$. As we already explained above $M / G^{T}=\mathbb{C}^{*}$, so the relative cohomology is non-zero only in degree 2 , i.e., for $i=2$. Since $M$ is a Stein manifold, we can describe the relative cohomology in terms of the holomorphic de Rham complexes on $\mathbb{C}^{2}$ and $M$. Namely, consider the complex of Abelian groups

$$
\Gamma\left(\mathbb{C}^{2}, \Omega_{\mathbb{C}^{2}}^{\bullet}\right)^{G^{T}} \oplus \Gamma\left(M, \Omega_{M}^{\bullet-1}\right)^{G^{T}}, \quad d(\omega, \alpha)=\left(d \omega,\left.\omega\right|_{M}-d \alpha\right) .
$$

A closed form $(\omega, \alpha)$ in degree $i$, that is, $d(\omega, \alpha)=0$, defines naturally a linear functional on the space of dimension $i$ relative chains $\gamma \subset \mathbb{C}^{2}$ with $\partial \gamma \subset M$, that is,

$$
\gamma \mapsto \int_{\gamma} \omega-\int_{\partial \gamma} \alpha
$$

Using the de Rham theorem for $\mathbb{C}^{2}$ and $M$, it is easy to prove that the above map induces an isomorphism between the $i$-th cohomology of the complex $(3.3)$ and $H^{i}\left(\mathbb{C}^{2}, M ; \mathbb{C}\right)^{G^{T}}$. Let us denote by $e_{N} \in H^{2}\left(\mathbb{C}^{2}, M ; \mathbb{C}\right)$ the cohomology class corresponding to the form $\left(0,-\frac{1}{2 \pi \mathbf{i}} d x_{1} / x_{1}\right)$.

Suppose now that $g=\left(-1, \eta^{2 a-1},-1\right), 1 \leq a \leq N-1$. Let us compute the component of $\operatorname{ch}_{\Gamma}\left(E_{i}^{\bullet}\right)$ for $1 \leq i \leq N-1$ in $H^{0}\left(\operatorname{Fix}_{g}\left(\mathbb{C}^{3}\right), \operatorname{Fix}_{g}\left(V^{T}\right) ; \mathbb{C}\right)^{G^{T}}$. Note that in this case we have an isomorphism $K^{0}\left(\operatorname{Fix}_{g}\left(\mathbb{C}^{3}\right), \operatorname{Fix}_{g}\left(V^{T}\right)\right) \cong K^{0}\left(\operatorname{Fix}_{g}\left(\mathbb{C}^{3}\right)\right)$. The image of $\iota^{*} \operatorname{Tr}\left(E_{i}^{\bullet}\right)$ is

$$
\eta^{-(2 a-1)(i-1)} L_{2}^{i-1}\left(L_{1} L_{3}-\left(-L_{3}-L_{1}\right)+\mathbb{C}\right)=4 \eta^{-(2 a-1)(i-1)} \mathbb{C},
$$

where again we abused the notation by denoting by $L_{i}$ the restriction of $L_{i}$ to $\operatorname{Fix}_{g}\left(\mathbb{C}^{3}\right)=\{0\}$. The component of the $\Gamma$-class is

$$
\Gamma\left(L_{1}+L_{2}+L_{3}\right)=\Gamma(1-1 / 2) \Gamma(1-(2 a-1) / h) \Gamma(1-1 / 2) e_{a},
$$

where $h:=2 N-2$. Therefore, the component of $\operatorname{ch}_{\Gamma}\left(E_{i}^{\bullet}\right)$ is

$$
2 \eta^{-(2 a-1)(i-1)} \Gamma\left(1-m_{a} / h\right) e_{a} .
$$

The component of $\operatorname{ch}_{\Gamma}\left(E_{N}\right)$ is clearly 0 , because the image of the complex $E_{N}^{\bullet}$ in $K^{0}\left(\operatorname{Fix}_{g}\left(\mathbb{C}^{3}\right)\right)$ is 0 .

Suppose that $g=(1,1,-1)$. Let us compute the component of $\operatorname{ch}_{\Gamma}\left(E_{i}^{\bullet}\right)$ in

$$
H^{2}\left(\operatorname{Fix}_{g}\left(\mathbb{C}^{3}\right), \operatorname{Fix}_{g}\left(V^{T}\right) ; \mathbb{C}\right)^{G^{T}}=H^{2}\left(\mathbb{C}^{2}, M\right)^{G^{T}}=\mathbb{C} e_{N}
$$

By definition the component of $\iota^{*} \operatorname{Tr}\left(E_{i}^{\bullet}\right)$ in $K^{0}\left(\mathbb{C}^{2}, M\right)^{G^{T}}$ is

$$
-L_{2}^{i-1}\left[L_{1} L_{3} \stackrel{-x_{1}}{\longrightarrow} L_{3} \longrightarrow 0\right]+L_{2}^{i-1}\left[0 \longrightarrow L_{1} \stackrel{x_{1}}{\longrightarrow} \mathbb{C}\right]
$$


where the above complexes are concentrated in degrees 0,1 , and 2 and the vector bundles $L_{i}$ $(1 \leq i \leq 3)$ are trivial line bundles on $\mathbb{C}^{2}$. The second complex, as an element of $K^{0}\left(\mathbb{C}^{2}, M\right)$,

is equivalent to the two-term complex $\underline{\mathbb{C}} \stackrel{\bar{x}_{1}}{\longrightarrow} \underline{\mathbb{C}}$. Therefore, the component of $\iota^{*} \operatorname{Tr}\left(E_{i}^{\bullet}\right)(1 \leq$ $i \leq N-1$ ) takes the form

$$
-\left[\underline{\mathbb{C}} \stackrel{x_{1}}{\longrightarrow} \mathbb{C}\right]+\left[\underline{\mathbb{C}} \stackrel{\bar{x}_{1}}{\longrightarrow} \mathbb{C}\right] .
$$

In order to compute the Chern character of the above complexes, we use the following commutative diagram:

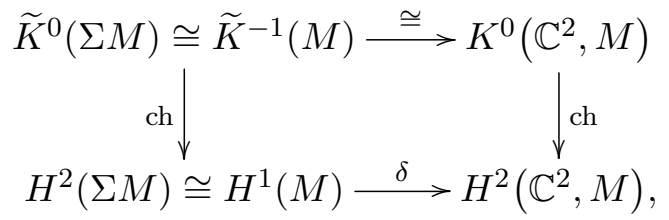

where the horizontal arrows come from the long exact sequence of the pair $\left(\mathbb{C}^{2}, M\right)$ and the vertical arrows are isomorphisms. Under the isomorphism $\widetilde{K}^{0}(\Sigma M) \cong K^{0}\left(\mathbb{C}^{2}, M\right)$, the complex $\underline{\mathbb{C}} \stackrel{x_{1}}{\longrightarrow} \mathbb{C}$ corresponds to $P-1$, where $P$ is a line bundle on $\Sigma M$ obtained by gluing two trivial line bundles along $M$ using the gluing function $M \rightarrow \mathbb{C}^{*},\left(x_{1}, x_{2}\right) \mapsto x_{1}$. The first Chern class of $P$ is easy to compute. If $\gamma$ is a closed loop in $M$ representing a cohomology class in $H_{1}(M)$, then $\Sigma \gamma$ is a sphere in $H_{2}(\Sigma M)$ and hence $\left.P\right|_{\Sigma \gamma}$ is a line bundle on the sphere obtained from gluing two trivial line bundles on the two hemi-spheres along the equator $\gamma$ using the map $\gamma \rightarrow \mathbb{C}^{*},\left(x_{1}, x_{2}\right) \rightarrow x_{1}$. By definition $\left\langle c_{1}(P), \Sigma \gamma\right\rangle$ coincides with the degree of the map $\gamma \rightarrow \mathbb{S}^{1}$, $\left(x_{1}, x_{2}\right) \mapsto x_{1} /\left|x_{1}\right|$, that is,

$$
\left\langle c_{1}(P), \Sigma \gamma\right\rangle=\frac{1}{2 \pi \mathbf{i}} \int_{\gamma} \frac{d x_{1}}{x_{1}} .
$$

In other words, under the suspension isomorphism, $c_{1}(P)$ coincides with the de Rham cohomology class of the form $\frac{1}{2 \pi \mathbf{i}} d x_{1} / x_{1}$. Recalling the de Rham model for the relative cohomology group $H^{2}\left(\mathbb{C}^{2}, M\right)$, we get that $\delta\left(c_{1}(P)\right)=e_{N}$. Note that $c_{1}(P)=\operatorname{ch}(P-1), \operatorname{so} \operatorname{ch}\left(\underline{\mathbb{C}} \stackrel{x_{1}}{\longrightarrow} \underline{\mathbb{C}}\right)=e_{N}$.

The vector bundle corresponding to the other complex $\underline{\mathbb{C}} \stackrel{\bar{x}_{1}}{\longrightarrow} \underline{\mathbb{C}}$ is $P^{-1}$, so we get

$$
\operatorname{ch}\left(\iota^{*} \operatorname{Tr}\left(E_{i}^{\bullet}\right)\right)=-2 e_{N} .
$$

Hence

$$
\operatorname{ch}_{\Gamma}\left(E_{i}^{\bullet}\right)_{g}=\frac{1}{2 \pi} \Gamma(1 / 2)(2 \pi \mathbf{i})\left(-2 e_{N}\right)=-2 \mathbf{i} \Gamma(1 / 2) e_{N},
$$

where the index $g$ is to remind us that this is the component corresponding to the fixed point set of $g=(1,1,-1)$. The computation of $\operatorname{ch}_{\Gamma}\left(E_{N}^{\bullet}\right)$ is the same, except that everywhere we have to replace the vector bundle $P$ with $P^{2}$, so

$$
\operatorname{ch}_{\Gamma}\left(E_{N}^{\bullet}\right)_{g}=\frac{1}{2 \pi} \Gamma(1 / 2)(2 \pi \mathbf{i})\left(-4 e_{N}\right)=-4 \mathbf{i} \Gamma(1 / 2) e_{N} .
$$

Combining our computations we get the following result:

$$
\operatorname{ch}_{\Gamma}\left(E_{i}^{\bullet}\right)=2 \sum_{a=1}^{N-1} \eta^{-(2 a-1)(i-1)} \Gamma\left(1-m_{a} / h\right) e_{a}-2 \mathbf{i} \Gamma(1 / 2) e_{N}
$$

and $\operatorname{ch}_{\Gamma}\left(E_{N}^{\bullet}\right)=-4 \mathbf{i} \Gamma(1 / 2) e_{N}$. Comparing with formula (2.6) we get that if we define $\operatorname{mir}\left(\phi_{i}\right)=e_{i}$ for $1 \leq i \leq N-1$ and $\operatorname{mir}\left(\phi_{N}\right)=2 e_{N}$, then the statement of Theorem 1.3 will hold. The vanishing cycle $\alpha_{k}(1 \leq k \leq N-1)$ corresponds to the relative $K$-theoretic class of the complex $E_{k}^{\bullet}$. 


\section{7 $\quad \Gamma$-integral structure for $E_{7}$-singularity}

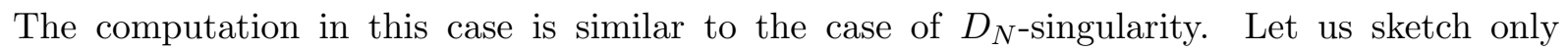
the main steps and leave the details as an exercise. The goal is to $\operatorname{compute} \mathrm{ch}_{\Gamma}\left(E_{l}^{\bullet}\right)$ for $1 \leq l \leq 7$. After a straightforward computation we get that the relative cohomology group $H\left(\operatorname{Fix}_{g}\left(\mathbb{C}^{3}\right), \operatorname{Fix}_{g}\left(V^{T}\right)\right)^{G^{T}}$ is not zero only in the following two cases: 1$) g=\left(g_{1}, g_{2}, g_{3}\right)$ with $g_{i} \neq 1$ for all $i$ and 2) $g=(1,1,-1)$. Put $\eta=e^{2 \pi \mathbf{i} / 9}$ and $\eta_{3}=e^{2 \pi \mathbf{i} / 3}$. For the first case, there are 6 elements, that is, $g=\left(\eta^{3 i-r}, \eta_{3}^{r},-1\right)(1 \leq i \leq 3,1 \leq r \leq 2)$ and the fixed-point subsets are $\operatorname{Fix}_{g}\left(\mathbb{C}^{3}\right)=\{0\}$ and $\operatorname{Fix}_{g}\left(V^{T}\right)=\varnothing$. Therefore, $H\left(\operatorname{Fix}_{g}\left(\mathbb{C}^{3}\right), \operatorname{Fix}_{g}\left(V^{T}\right) ; \mathbb{C}\right)^{G^{T}} \cong \mathbb{C}$ is nontrivial only in degree 0 and we denote by $e_{3 i-r} \in H\left(\operatorname{Fix}_{g}\left(\mathbb{C}^{3}\right), \operatorname{Fix}_{g}\left(V^{T}\right) ; \mathbb{C}\right)^{G^{T}}$ the unit of the cohomology group. For the second case, $\operatorname{Fix}_{g}\left(\mathbb{C}^{3}\right)=\mathbb{C}^{2}$ and $\operatorname{Fix}_{g}\left(V^{T}\right)=M=\left\{x_{1}^{3} x_{2}+x_{2}^{3}=1\right\}$. The relative cohomology group $H^{i}\left(\mathbb{C}^{2}, M ; \mathbb{C}\right)^{G^{T}} \cong H^{i-1}\left(M / G^{T} ; \mathbb{C}\right)$ for $i>0$ and $=0$ for $i=0$. Just like in the $D_{N^{-}}$case, we have $M / G^{T}=\mathbb{C}^{*}$, so the relative cohomology is non-zero only in degree 2 , i.e., for $i=2$. Let us denote by $e_{7} \in H^{2}\left(\mathbb{C}^{2}, M\right)^{G^{T}}$ the cohomology class corresponding to the differential form $\left(0,-\frac{1}{2 \pi i} d x_{2} / x_{2}\right)$.

Suppose that $g=\left(\eta^{3 i-r}, \eta_{3}^{r},-1\right)$. We have

$$
\iota^{*} \operatorname{Tr}\left(E_{l}^{\bullet}\right)_{g}=-2\left(1-\eta_{3}^{-r}\right) \eta^{-(3 i-r)(l-1)} \mathbb{C} \in K^{0}\left(\operatorname{Fix}_{g}\left(\mathbb{C}^{3}\right)\right)
$$

and the component of the $\Gamma$-class of $\left[T \mathbb{C}^{3} / G^{T}\right]$ in $H\left(\operatorname{Fix}_{g}\left(\mathbb{C}^{3}\right) / G^{T}\right)$ is

$$
\Gamma(1-(3 i-r) / 9) \Gamma(1-r / 3) \Gamma(1 / 2) \text {. }
$$

Therefore

$$
\operatorname{ch}_{\Gamma}\left(E_{l}^{\bullet}\right)_{g}=-\frac{1}{\sqrt{\pi}}\left(1-\eta_{3}^{-r}\right) \eta^{-(3 i-r)(l-1)} \Gamma(1-(3 i-r) / 9) \Gamma(1-r / 3) e_{3 i-r}, \quad 1 \leq l \leq 6 .
$$

Note that $\operatorname{ch}_{\Gamma}\left(E_{7}^{\bullet}\right)_{g}=0$.

Suppose now that $g=(1,1,-1)$. Then we have

$$
\iota^{*} \operatorname{Tr}\left(E_{l}^{\bullet}\right)_{g}=-\left[\underline{\mathbb{C}} \stackrel{x_{2}}{\longrightarrow} \underline{\mathbb{C}} \longrightarrow 0\right]+\left[0 \longrightarrow \underline{\mathbb{C}} \stackrel{x_{2}}{\longrightarrow} \underline{\mathbb{C}}\right] .
$$

Under the isomorphism $\widetilde{K}^{0}(\Sigma M) \cong K^{0}\left(\mathbb{C}^{2}, M\right)$, the complex $\left[\underline{\mathbb{C}} \stackrel{x_{2}}{\longrightarrow} \underline{\mathbb{C}}\right]$ corresponds to $P-1$, where $P$ is a vector bundle on $\Sigma M$ obtained by gluing two trivial line bundles along $M$ with gluing function $M \rightarrow \mathbb{C}^{*},\left(x_{1}, x_{2}\right) \mapsto x_{2}$. Under the suspension isomorphism $H^{2}(\Sigma M) \cong H^{1}(M)$, we have that $\operatorname{ch}(P-1)=c_{1}(P)$ is the cohomology class corresponding to the form $\frac{1}{2 \pi \mathbf{i}} d x_{2} / x_{2}$. The latter, under the boundary isomorphism $H^{1}(M) \rightarrow H^{2}\left(\mathbb{C}^{2}, M\right)$ is mapped precisely to $e_{7}$, that is, $\operatorname{ch}\left(\left[\underline{\mathbb{C}} \stackrel{x_{2}}{\longrightarrow} \mathbb{C}\right]\right)=e_{7}$. The Chern character of the second complex in $(3.4)$ is $-e_{7}$, so we get $\operatorname{ch}\left(\iota^{*} \operatorname{Tr}\left(E_{l}^{\bullet}\right)\right)_{g}=-2 e_{7}$. Hence

$$
\operatorname{ch}_{\Gamma}\left(E_{l}^{\bullet}\right)_{g}=\frac{1}{2 \pi} \Gamma(1 / 2)(2 \pi \mathbf{i})\left(-2 e_{7}\right)=-2 \mathbf{i} \sqrt{\pi} e_{7} .
$$

The computation of $\operatorname{ch}_{\Gamma}\left(E_{7}^{\bullet}\right)_{g}$ is the same as above except that we have to replace the bundle $P$ with $P^{3}$, that is, we get $\operatorname{ch}_{\Gamma}\left(E_{7}^{\bullet}\right)_{g}=-6 \mathbf{i} \sqrt{\pi} e_{7}$.

Collecting the results of our computations we get

$$
\operatorname{ch}_{\Gamma}\left(E_{l}^{\bullet}\right)=-\sum_{r=1}^{2} \sum_{i=1}^{3} \frac{1}{\sqrt{\pi}}\left(1-\eta_{3}^{-r}\right) \eta^{-(3 i-r)(l-1)} \Gamma(1-(3 i-r) / 9) \Gamma(1-r / 3) e_{3 i-r}-2 \mathbf{i} \sqrt{\pi} e_{7}
$$


for $1 \leq l \leq 6$ and $\operatorname{ch}_{\Gamma}\left(E_{7}^{\bullet}\right)=-6 \mathbf{i} \sqrt{\pi} e_{7}$. Let us compare the above formula with (2.8). Note that $1-\eta_{3}^{-1}=\sqrt{3} e^{\pi \mathbf{i} / 6}$ and $1-\eta_{3}^{-2}=\sqrt{3} e^{-\pi \mathbf{i} / 6}$. We would like to find $k, a \in \mathbb{Z}_{3}$, such that, $\operatorname{mir} \circ \Psi\left(\alpha_{k, a}\right)=\operatorname{ch}_{\Gamma}\left(E_{l}^{\bullet}\right)$. Let us write $l-1=3 m+k$ for $0 \leq k \leq 2,0 \leq m \leq 1$. Then the above formula will hold if we choose $a=-m$ and define

$$
\operatorname{mir}\left(\phi_{i}\right)=e_{3 i-1}, \quad 1 \leq i \leq 3, \quad \operatorname{mir}\left(\phi_{j+3}\right)=e_{3 j-2}, \quad 1 \leq j \leq 3, \quad \operatorname{mir}\left(\phi_{7}\right)=-e_{7} .
$$

Note that mir $\circ \Psi\left(\alpha_{k, 0}+\alpha_{k, 1}+\alpha_{k, 2}\right)=\operatorname{ch}_{\Gamma}\left(E_{7}^{\bullet}\right)$. Using these formulas, we get immediately that the maps mir $\circ \Psi$ and $\mathrm{ch}_{\Gamma}$ identify the Milnor lattice $H_{2}\left(f^{-1}(1) ; \mathbb{Z}\right)$ with the relative K-ring $K^{0}\left(\mathbb{C}^{3}, V^{T}\right)$. This completes the proof of Theorem 1.3.

\section{Acknowledgements}

The work of T.M. is partially supported by JSPS Grant-in-Aid (Kiban C) 17K05193 and by the World Premier International Research Center Initiative (WPI Initiative), MEXT, Japan. The work of C.Z. is supported by JSPS DC1 program including Grant-in-Aid and was previously supported by MEXT scholarship. We would like to thank the referees to our paper for pointing out several inaccuracies and useful suggestions.

\section{References}

[1] Arnol'd V.I., Guseřn-Zade S.M., Varchenko A.N., Singularities of differentiable maps, Vol. II. Monodromy and asymptotics of integrals, Monographs in Mathematics, Vol. 83, Birkhäuser Boston, Inc., Boston, MA, 1988.

[2] Atiyah M., Segal G., On equivariant Euler characteristics, J. Geom. Phys. 6 (1989), 671-677.

[3] Berglund P., Hübsch T., A generalized construction of mirror manifolds, Nuclear Phys. B 393 (1993), 377-391, arXiv:hep-th/9201014.

[4] Chiodo A., Iritani H., Ruan Y., Landau-Ginzburg/Calabi-Yau correspondence, global mirror symmetry and Orlov equivalence, Publ. Math. Inst. Hautes Études Sci. 119 (2014), 127-216, arXiv:1201.0813.

[5] Chiodo A., Nagel J., The hybrid Landau-Ginzburg models of Calabi-Yau complete intersections, in Topological Recursion and its Influence in Analysis, Geometry, and Topology, Proc. Sympos. Pure Math., Vol. 100, Amer. Math. Soc., Providence, RI, 2018, 103-117, arXiv:1506.02989.

[6] Fan H., Jarvis T., Ruan Y., The Witten equation, mirror symmetry, and quantum singularity theory, Ann. of Math. 178 (2013), 1-106, arXiv:0712.4021.

[7] Frenkel E., Givental A., Milanov T., Soliton equations, vertex operators, and simple singularities, Funct. Anal. Other Math. 3 (2010), 47-63, arXiv:0909.4032.

[8] Givental A.B., Gromov-Witten invariants and quantization of quadratic Hamiltonians, Mosc. Math. J. 1 (2001), 551-568, arXiv:math.AG/0108100.

[9] Givental A.B., Semisimple Frobenius structures at higher genus, Int. Math. Res. Not. 2001 (2001), 12651286, arXiv:math.AG/0008067.

[10] Givental A.B., Milanov T.E., Simple singularities and integrable hierarchies, in The Breadth of Symplectic and Poisson Geometry, Progr. Math., Vol. 232, Birkhäuser Boston, Boston, MA, 2005, 173-201, arXiv:math.AG/0307176.

[11] Hertling C., Frobenius manifolds and moduli spaces for singularities, Cambridge Tracts in Mathematics, Vol. 151, Cambridge University Press, Cambridge, 2002.

[12] Iritani H., An integral structure in quantum cohomology and mirror symmetry for toric orbifolds, Adv. Math. 222 (2009), 1016-1079, arXiv:0903.1463.

[13] Kontsevich M., Intersection theory on the moduli space of curves and the matrix Airy function, Comm. Math. Phys. 147 (1992), 1-23.

[14] Krawitz M., FJRW rings and Landau-Ginzburg mirror symmetry, Ph.D. Thesis, University of Michigan, 2010.

[15] Minami H., A Künneth formula for equivariant K-theory, Osaka Math. J. 6 (1969), 143-146. 
[16] Saito K., On periods of primitive integrals, I, Preprint, Research Institute for Mathematical Sciences, Kyoto University, 1982.

[17] Satake I., On a generalization of the notion of manifold, Proc. Nat. Acad. Sci. USA 42 (1956), 359-363.

[18] Segal G., Equivariant K-theory, Inst. Hautes Études Sci. Publ. Math. (1968), 129-151.

[19] Teleman C., The structure of 2D semi-simple field theories, Invent. Math. 188 (2012), 525-588, arXiv:0712.0160. 